\title{
INFLUENCE OF STELLAR MULTIPLICITY ON PLANET FORMATION. IV. ADAPTIVE OPTICS IMAGING OF KEPLER STARS WITH MULTIPLE TRANSITING PLANET CANDIDATES
}

\author{
Ji Wang ${ }^{1,2}$, Debra A. Fischer ${ }^{1}$, Ji$_{\text {-Wei }} \mathrm{XIE}^{3}$, AND David R. Ciardi ${ }^{4}$ \\ ${ }^{1}$ Department of Astronomy, Yale University, New Haven, CT 06511, USA; ji.wang@yale.edu \\ 2 Department of Astronomy, Califonia Institute of Technology, MC 249-17, 1200 East California, Blvd., Pasadena, CA 91125 USA \\ ${ }^{3}$ Department of Astronomy \& Key Laboratory of Modern Astronomy and Astrophysics in Ministry of Education, Nanjing University, Nanjing 210093, China \\ ${ }^{4}$ NASA Exoplanet Science Institute, Caltech, MS 100-22, 770 South Wilson Avenue, Pasadena, CA 91125, USA \\ Received 2015 August 5; accepted 2015 October 6; published 2015 November 6
}

\begin{abstract}
The Kepler mission provides a wealth of multiple transiting planet systems (MTPSs). The formation and evolution of multi-planet systems are likely to be influenced by companion stars given the abundance of multiple stellar systems. We study the influence of stellar companions by measuring the stellar multiplicity rate of MTPSs. We select 138 bright $\left(K_{P}<13.5\right)$ Kepler MTPSs and search for stellar companions with adaptive optics (AO) imaging data and archival radial velocity data. We obtain new AO images for 73 MTPSs. Other MTPSs in the sample have archival AO imaging data from the Kepler Community Follow-up Observation Program. From these imaging data, we detect 42 stellar companions around 35 host stars. For stellar separation $1 \mathrm{AU}<a<100 \mathrm{AU}$, the stellar multiplicity rate is $5.2 \pm 5.0 \%$ for MTPSs, which is $2.8 \sigma$ lower than $21.1 \pm 2.8 \%$ for the control sample, i.e., the field stars in the solar neighborhood. We identify two origins for the deficit of stellar companions within $100 \mathrm{AU}$ of MTPSs: (1) a suppressive planet formation and (2) the disruption of orbital coplanarity due to stellar companions. To distinguish between the two origins, we compare the stellar multiplicity rates of MTPSs and single transiting planet systems (STPSs). However, current data are not sufficient for this purpose. For $100 \mathrm{AU}<a<2000 \mathrm{AU}$, the stellar multiplicity rates are comparable for MTPSs $(8.0 \pm 4.0 \%)$, STPSs $(6.4 \pm 5.8 \%)$, and the control sample $(12.5 \pm 2.8 \%)$.
\end{abstract}

Key words: methods: observational - planet-star interactions - planetary systems planets and satellites: dynamical evolution and stability - planets and satellites: formation techniques: high angular resolution

\section{INTRODUCTION}

As exoplanet surveys reach higher sensitivity and a longer time baseline, more exoplanets are being discovered. Many of these exoplanets are in multi-planet systems. As of 2015 September, the radial velocity (RV) technique and the transit method have detected 152 and 857 planets in multi-planet systems (http:// exoplanets.org; Han et al. 2014). From these systems, we can study their orbital spacing (e.g., Wright et al. 2011; Burke et al. 2014), mutual inclination (e.g., Lissauer et al. 2011; Tremaine \& Dong 2012), and eccentricity distribution (e.g., Jurić \& Tremaine 2008; Kane et al. 2012; J.-W. Xie 2015, in preparation). These studies can be used to test theories of planet formation and dynamical evolution (Winn \& Fabrycky 2015).

While only $\sim 20 \%$ of Kepler planet host stars are multiple transiting planet systems (MTPSs), the total number of planets in an MTPS accounts for almost half of the Kepler planet candidates. Latham et al. (2011) compared Kepler MTPSs to single transiting planet systems (STPSs). They found a lack of gas giant planets in MTPSs, which indicates that the existence of a gas giant planet may disrupt the orbital inclinations or suppress the formation of multiple planets. Furthermore, other studies implied that the distributions of orbital spacings (Xie et al. 2014), eccentricities (J.-W. Xie 2015, in preparation), and obliquities (Morton \& Winn 2014) are different for STPSs and MTPSs. In this paper, we investigate one possibility that causes the different orbital architecture between STPSs and MTPSs, namely, the influence of dynamically bound companion stars.

By comparing stellar multiplicity rate for 138 MTPSs against stars in the solar neighborhood (Duquennoy \& Mayor 1991; Raghavan et al. 2010), Wang et al. (2014b) found evidence of suppressive planet formation in multiple stellar systems with stellar separations smaller than $20 \mathrm{AU}$. Beyond $20 \mathrm{AU}$, the stellar multiplicity rate was difficult to measure without high-resolution and deep-imaging data that provide sensitivity to stellar companions at these separations. Therefore, at separations wider than $20 \mathrm{AU}$, the influence of stellar companions on multi-planet formation was not well understood. In this paper, we gather adaptive optics (AO) images for the same MTPS sample in Wang et al. (2014b). Since AO images for 65 MTPSs are already available from the Kepler Community Follow-up Observation Program $^{4}$ (CFOP), we obtain new AO images for the remaining 73 MTPSs at Keck observatory and Palomar observatory. The archival and newly obtained AO images reveal dozens of new stellar companions to planet host stars and put valuable constraints on multi-planet formation in multiple stellar systems.

The paper is organized as follows. We describe the sample selection and $\mathrm{AO}$ data acquisition in Section 2, followed by data analyses in Section 3. We report the stellar multiplicity rate for MTPSs in Section 4. Discussion and summary are given in Section 5.

\section{SAMPLE DESCRIPTION AND AO DATA ACQUISITION}

\subsection{Sample Description}

The sample of MTPSs remains the same as that in Wang et al. (2014b). From the NASA Exoplanet Archive ${ }^{5}$, we select

\footnotetext{
4 https://cfop.ipac.caltech.edu

5 http://exoplanetarchive.ipac.caltech.edu
} 
Table 1

AO Sensitivity

\begin{tabular}{|c|c|c|c|c|c|c|c|c|c|c|c|c|c|c|c|c|}
\hline \multicolumn{7}{|c|}{ Kepler } & \multicolumn{4}{|c|}{ Observation } & \multicolumn{6}{|c|}{ 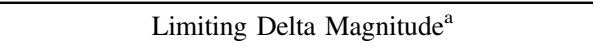 } \\
\hline KIC & KOI & $\begin{array}{l}\text { Kmag } \\
(\mathrm{mag})\end{array}$ & $\begin{array}{c}i \\
(\mathrm{mag})\end{array}$ & $\begin{array}{c}J \\
(\mathrm{mag})\end{array}$ & $\begin{array}{c}H \\
(\mathrm{mag})\end{array}$ & $\begin{array}{c}K \\
(\mathrm{mag})\end{array}$ & $\begin{array}{c}\text { Companion } \\
\text { within 5" }\end{array}$ & $\begin{array}{c}\text { Isolation } \\
\text { Probability }^{\mathrm{b}}\end{array}$ & Instrument & $\overline{\text { Filter }}$ & $\begin{array}{l}0.1 \\
(")\end{array}$ & $\begin{array}{l}0.2 \\
\left({ }^{\prime \prime}\right)\end{array}$ & $\begin{array}{l}0.5 \\
\left({ }^{\prime \prime}\right)\end{array}$ & $\begin{array}{l}1.0 \\
(")\end{array}$ & $\begin{array}{l}2.0 \\
(\prime)\end{array}$ & $\begin{array}{l}4.0 \\
\left({ }^{\prime \prime}\right)\end{array}$ \\
\hline 8554498 & 00005 & 11.665 & 11.485 & 10.542 & 10.257 & 10.213 & yes & $\ldots$ & NIRC2 & $K$ & 2.0 & 4.0 & 6.4 & 7.4 & 7.5 & $\overline{7.5}$ \\
\hline 8554498 & 00005 & 11.665 & 11.485 & 10.542 & 10.257 & 10.213 & yes & $\ldots$ & PHARO & $J$ & 0.1 & 1.3 & 2.7 & 4.8 & 6.7 & 7.6 \\
\hline 6521045 & 00041 & 11.197 & 11.030 & 10.081 & 9.804 & 9.768 & no & 0.96 & ARIES & $K$ & 0.1 & 1.2 & 3.4 & 5.9 & 7.2 & 7.5 \\
\hline 6521045 & 00041 & 11.197 & 11.030 & 10.081 & 9.804 & 9.768 & no & 0.96 & NIRC2 & $K$ & 3.2 & 4.6 & 5.3 & 5.4 & 5.4 & 5.4 \\
\hline 6521045 & 00041 & 11.197 & 11.030 & 10.081 & 9.804 & 9.768 & no & 0.96 & PHARO & $J$ & 0.4 & 3.6 & 4.5 & 6.6 & 7.6 & 7.7 \\
\hline 6850504 & 00070 & 12.498 & 12.284 & 11.252 & 10.910 & 10.871 & yes & $\ldots$ & PHARO & $J$ & 0.4 & 3.0 & 4.5 & 6.4 & 7.3 & 7.4 \\
\hline 11904151 & 00072 & 10.961 & 10.778 & 9.889 & 9.563 & 9.496 & no & 0.99 & ARIES & $K$ & 0.8 & 2.3 & 5.1 & 7.0 & 7.6 & 7.6 \\
\hline 11904151 & 00072 & 10.961 & 10.778 & 9.889 & 9.563 & 9.496 & no & 0.99 & PHARO & $J$ & 0.5 & 3.3 & 4.3 & 6.5 & 7.9 & 8.1 \\
\hline 10187017 & 00082 & 11.492 & 11.150 & 9.984 & 9.446 & 9.351 & no & 0.92 & ARIES & $K$ & 0.5 & 1.9 & 4.7 & 7.0 & 7.8 & 7.9 \\
\hline 10187017 & 00082 & 11.492 & 11.150 & 9.984 & 9.446 & 9.351 & no & 0.92 & NIRC2 & $K$ & 2.6 & 4.5 & 5.4 & 5.6 & 5.6 & 5.6 \\
\hline 5866724 & 00085 & 11.018 & 10.882 & 10.066 & 9.852 & 9.806 & no & 0.88 & ARIES & $K$ & 0.9 & 2.4 & 5.1 & 7.1 & 7.6 & 7.7 \\
\hline 6462863 & 00094 & 12.205 & 12.057 & 11.218 & 10.957 & 10.926 & no & 0.75 & ARIES & $K$ & 0.1 & 0.9 & 4.2 & 6.8 & 7.4 & 7.3 \\
\hline 8456679 & 00102 & 12.566 & 12.384 & 11.398 & 11.124 & 11.055 & yes & $\ldots$ & NIRC2 & $K$ & 2.2 & 4.3 & 6.3 & 7.2 & 7.3 & 7.3 \\
\hline 8456679 & 00102 & 12.566 & 12.384 & 11.398 & 11.124 & 11.055 & yes & $\ldots$ & PHARO & $J$ & 0.7 & 2.2 & 4.0 & 5.8 & 6.9 & 7.4 \\
\hline 4914423 & 00108 & 12.287 & 12.132 & 11.193 & 10.941 & 10.873 & yes & $\ldots$ & NIRC2 & $K$ & 2.5 & 4.0 & 5.7 & 6.1 & 6.2 & 6.2 \\
\hline 4914423 & 00108 & 12.287 & 12.132 & 11.193 & 10.941 & 10.873 & yes & $\ldots$ & PHARO & $J$ & 0.8 & 3.2 & 4.4 & 6.5 & 7.6 & 7.7 \\
\hline 6678383 & 00111 & 12.596 & 12.442 & 11.558 & 11.251 & 11.209 & no & 0.89 & PHARO & $J$ & 0.6 & 2.9 & 4.2 & 6.1 & 7.1 & 7.3 \\
\hline 10984090 & 00112 & 12.772 & 12.602 & 11.698 & 11.402 & 11.367 & no & 0.84 & PHARO & $J$ & 0.5 & 2.4 & 3.9 & 6.1 & 8.0 & 8.5 \\
\hline 10984090 & 00112 & 12.772 & 12.602 & 11.698 & 11.402 & 11.367 & no & 0.84 & PHARO & $K$ & 0.0 & 1.8 & 4.8 & 5.4 & 6.9 & 7.1 \\
\hline 9579641 & 00115 & 12.791 & 12.654 & 11.811 & 11.555 & 11.503 & yes & & ARIES & $K$ & 0.2 & 1.8 & 4.9 & 6.6 & 6.8 & 6.8 \\
\hline 8395660 & 00116 & 12.882 & 12.706 & 11.752 & 11.494 & 11.431 & no & 0.91 & ARIES & $K$ & 0.4 & 1.9 & 4.9 & 7.0 & 7.3 & 7.2 \\
\hline 8395660 & 00116 & 12.882 & 12.706 & 11.752 & 11.494 & 11.431 & no & 0.91 & NIRC2 & $K$ & 2.9 & 4.5 & 6.2 & 6.5 & 6.6 & 6.6 \\
\hline 10875245 & 00117 & 12.487 & 12.309 & 11.392 & 11.114 & 11.060 & no & 0.74 & PHARO & $J$ & 0.1 & 0.7 & 2.1 & 3.7 & 5.9 & 7.9 \\
\hline 10875245 & 00117 & 12.487 & 12.309 & 11.392 & 11.114 & 11.060 & no & 0.74 & PHARO & $K$ & 0.4 & 1.5 & 3.6 & 5.0 & 6.8 & 7.2 \\
\hline 9471974 & 00119 & 12.654 & 12.452 & 11.430 & 11.065 & 10.983 & yes & $\ldots$ & PHARO & $J$ & 0.0 & 0.6 & 1.8 & 3.3 & 4.5 & 7.3 \\
\hline 9471974 & 00119 & 12.654 & 12.452 & 11.430 & 11.065 & 10.983 & yes & $\ldots$ & PHARO & $K$ & 0.0 & 0.7 & 2.7 & 4.3 & 5.5 & 6.5 \\
\hline 5094751 & 00123 & 12.365 & 12.206 & 11.314 & 11.046 & 11.001 & no & 0.86 & NIRC2 & $K$ & 2.4 & 4.3 & 6.0 & 6.5 & 6.5 & 6.5 \\
\hline 5094751 & 00123 & 12.365 & 12.206 & 11.314 & 11.046 & 11.001 & no & 0.86 & PHARO & $J$ & 0.0 & 1.2 & 3.3 & 5.3 & 7.0 & 7.6 \\
\hline 5735762 & 00148 & 13.040 & 12.761 & 11.702 & 11.292 & 11.221 & yes & $\ldots$ & NIRC2 & $K$ & 2.3 & 4.2 & 5.7 & 6.3 & 6.3 & 6.3 \\
\hline 5735762 & 00148 & 13.040 & 12.761 & 11.702 & 11.292 & 11.221 & yes & $\ldots$ & PHARO & $J$ & 0.2 & 2.8 & 4.0 & 6.1 & 7.4 & 7.6 \\
\hline 12252424 & 00153 & 13.461 & 13.097 & 11.886 & 11.360 & 11.255 & no & 0.93 & ARIES & $K$ & 0.0 & 1.0 & 4.1 & 6.4 & 6.7 & 6.7 \\
\hline 12252424 & 00153 & 13.461 & 13.097 & 11.886 & 11.360 & 11.255 & no & 0.93 & NIRC2 & $K$ & 2.0 & 4.2 & 4.9 & 4.9 & 4.9 & 4.9 \\
\hline 12252424 & 00153 & 13.461 & 13.097 & 11.886 & 11.360 & 11.255 & no & 0.93 & PHARO & $J$ & 0.1 & 0.9 & 2.2 & 3.9 & 6.1 & 7.6 \\
\hline 12252424 & 00153 & 13.461 & 13.097 & 11.886 & 11.360 & 11.255 & no & 0.93 & PHARO & $K$ & 0.5 & 1.7 & 3.7 & 5.0 & 6.6 & 6.8 \\
\hline 11512246 & 00168 & 13.438 & 13.244 & 12.353 & 12.047 & 11.998 & no & 0.69 & PHARO & $K$ & 0.4 & 1.4 & 3.3 & 4.8 & 5.5 & 5.6 \\
\hline 4349452 & 00244 & 10.734 & $\ldots$ & 9.764 & 9.532 & 9.493 & no & 0.91 & NIRC2 & $K$ & 2.8 & 4.4 & 5.3 & 5.4 & 5.4 & 5.4 \\
\hline 4349452 & 00244 & 10.734 & $\ldots$ & 9.764 & 9.532 & 9.493 & no & 0.91 & PHARO & $J$ & 0.6 & 2.7 & 3.9 & 5.8 & 7.9 & 8.5 \\
\hline 4349452 & 00244 & 10.734 & $\ldots$ & 9.764 & 9.532 & 9.493 & no & 0.91 & PHARO & $K$ & 0.8 & 2.7 & 5.0 & 5.6 & 7.7 & 8.1 \\
\hline 8478994 & 00245 & 9.705 & $\ldots$ & 8.356 & 8.000 & 7.942 & no & 0.95 & ARIES & $K$ & 0.5 & 1.8 & 4.8 & 7.3 & 8.2 & 8.4 \\
\hline 8478994 & 00245 & 9.705 & $\ldots$ & 8.356 & 8.000 & 7.942 & no & 0.95 & NIRC2 & $K$ & 2.4 & 4.1 & 6.1 & 6.7 & 6.9 & 6.9 \\
\hline 8478994 & 00245 & 9.705 & $\ldots$ & 8.356 & 8.000 & 7.942 & no & 0.95 & PHARO & $K$ & 1.0 & 2.3 & 5.0 & 6.7 & 8.6 & 9.9 \\
\hline 11295426 & 00246 & 9.997 & 9.820 & 8.975 & 8.662 & 8.588 & no & 0.97 & ARIES & $K$ & 0.6 & 2.0 & 4.4 & 6.8 & 7.7 & 7.8 \\
\hline 11295426 & 00246 & 9.997 & 9.820 & 8.975 & 8.662 & 8.588 & no & 0.97 & NIRC2 & $K$ & 2.9 & 4.4 & 6.0 & 6.3 & 6.4 & 6.4 \\
\hline 8292840 & 00260 & 10.500 & $\ldots$ & 9.616 & 9.407 & 9.344 & no & 0.92 & ARIES & $K$ & 0.1 & 1.5 & 3.7 & 6.2 & 7.7 & 8.2 \\
\hline 11807274 & 00262 & 10.421 & 10.313 & 9.518 & 9.250 & 9.197 & no & 0.89 & ARIES & $K$ & 0.7 & 2.5 & 4.8 & 6.8 & 7.3 & 7.5 \\
\hline 6528464 & 00270 & 11.411 & $\ldots$ & 10.088 & 9.770 & 9.701 & no & 0.80 & ARIES & $K$ & 0.2 & 1.7 & 4.0 & 6.1 & 7.0 & 7.1 \\
\hline
\end{tabular}


Table 1

\begin{tabular}{|c|c|c|c|c|c|c|c|c|c|c|c|c|c|c|c|c|}
\hline \multicolumn{7}{|c|}{ Kepler } & \multicolumn{4}{|c|}{ Observation } & \multicolumn{6}{|c|}{ Limiting Delta Magnitude $^{\mathrm{a}}$} \\
\hline KIC & KOI & $\begin{array}{l}\text { Kmag } \\
(\mathrm{mag})\end{array}$ & $\begin{array}{c}i \\
(\mathrm{mag}) \\
\end{array}$ & $\begin{array}{c}J \\
(\mathrm{mag}) \\
\end{array}$ & $\begin{array}{c}H \\
(\mathrm{mag}) \\
\end{array}$ & $\begin{array}{c}K \\
(\mathrm{mag}) \\
\end{array}$ & $\begin{array}{c}\text { Companion } \\
\text { within } 5^{\prime \prime} \\
\end{array}$ & $\begin{array}{c}\text { Isolation } \\
\text { Probability }^{\mathrm{b}} \\
\end{array}$ & Instrument & Filter & $\begin{array}{l}0.1 \\
\left({ }^{\prime \prime}\right) \\
\end{array}$ & $\begin{array}{l}0.2 \\
(\prime)\end{array}$ & $\begin{array}{l}0.5 \\
(\prime)\end{array}$ & $\begin{array}{l}1.0 \\
\left({ }^{\prime \prime}\right) \\
\end{array}$ & $\begin{array}{l}2.0 \\
(\prime)\end{array}$ & $\begin{array}{l}4.0 \\
(") \\
\end{array}$ \\
\hline 9451706 & 00271 & 11.485 & 11.358 & 10.536 & 10.300 & 10.234 & no & 0.90 & ARIES & $K$ & 0.7 & 2.3 & 4.6 & 6.8 & 7.2 & $\overline{7.4}$ \\
\hline 9451706 & 00271 & 11.485 & 11.358 & 10.536 & 10.300 & 10.234 & no & 0.90 & NIRC2 & $K$ & 2.7 & 4.5 & 6.6 & 7.4 & 7.5 & 7.5 \\
\hline 9451706 & 00271 & 11.485 & 11.358 & 10.536 & 10.300 & 10.234 & no & 0.90 & PHARO & $J$ & 0.7 & 2.4 & 4.5 & 5.5 & 7.5 & 7.8 \\
\hline 9451706 & 00271 & 11.485 & 11.358 & 10.536 & 10.300 & 10.234 & no & 0.90 & PHARO & $K$ & 0.0 & 0.9 & 2.4 & 4.1 & 5.4 & 5.8 \\
\hline 8077137 & 00274 & 11.390 & 11.258 & 10.373 & 10.094 & 10.109 & no & 0.88 & ARIES & $K$ & 0.7 & 2.4 & 5.2 & 7.1 & 7.6 & 7.7 \\
\hline 10586004 & 00275 & 11.696 & $\ldots$ & 10.600 & 10.325 & 10.252 & no & 0.86 & PHARO & $J$ & 1.2 & 2.7 & 5.2 & 5.9 & 7.5 & 7.7 \\
\hline 10586004 & 00275 & 11.696 & $\ldots$ & 10.600 & 10.325 & 10.252 & no & 0.86 & PHARO & $K$ & 0.5 & 2.5 & 3.7 & 5.9 & 8.0 & 8.7 \\
\hline 12314973 & 00279 & 11.684 & 11.563 & 10.708 & 10.472 & 10.429 & yes & $\ldots$ & NIRC2 & $K$ & 2.1 & 4.3 & 5.5 & 5.6 & 5.7 & 5.7 \\
\hline 5088536 & 00282 & 11.529 & $\ldots$ & 10.810 & 10.529 & 10.490 & yes & $\ldots$ & NIRC2 & $K$ & 2.4 & 4.3 & 6.6 & 7.4 & 7.5 & 7.5 \\
\hline 5088536 & 00282 & 11.529 & $\ldots$ & 10.810 & 10.529 & 10.490 & yes & $\ldots$ & PHARO & $K$ & 0.5 & 1.5 & 3.6 & 5.7 & 7.3 & 7.6 \\
\hline 5695396 & 00283 & 11.525 & 11.334 & 10.418 & 10.127 & 10.079 & no & 0.95 & NIRC2 & $K$ & 2.5 & 3.9 & 5.2 & 5.5 & 5.5 & 5.5 \\
\hline 5695396 & 00283 & 11.525 & 11.334 & 10.418 & 10.127 & 10.079 & no & 0.95 & PHARO & $J$ & 0.0 & 0.5 & 1.7 & 3.1 & 5.2 & 7.4 \\
\hline 5695396 & 00283 & 11.525 & 11.334 & 10.418 & 10.127 & 10.079 & no & 0.95 & PHARO & $K$ & 0.8 & 2.2 & 4.1 & 5.8 & 7.3 & 7.7 \\
\hline 6021275 & 00284 & 11.818 & 11.666 & 10.797 & 10.516 & 10.424 & yes & $\ldots$ & PHARO & $J$ & 0.0 & 0.2 & 1.7 & 3.2 & 4.6 & 5.8 \\
\hline 6021275 & 00284 & 11.818 & 11.666 & 10.797 & 10.516 & 10.424 & yes & $\ldots$ & PHARO & $K$ & 0.0 & 0.4 & 1.6 & 3.7 & 5.1 & 7.9 \\
\hline 6196457 & 00285 & 11.565 & $\ldots$ & 10.747 & 10.470 & 10.403 & yes & $\ldots$ & PHARO & $J$ & 0.0 & 0.7 & 2.1 & 3.9 & 5.9 & 7.0 \\
\hline 6196457 & 00285 & 11.565 & $\ldots$ & 10.747 & 10.470 & 10.403 & yes & $\ldots$ & PHARO & $K$ & 0.4 & 1.9 & 3.9 & 5.6 & 7.1 & 7.5 \\
\hline 10386922 & 00289 & 12.747 & 12.540 & 11.534 & 11.220 & 11.187 & no & 0.92 & NIRC2 & $K$ & 2.5 & 4.5 & 6.5 & 7.2 & 7.3 & 7.3 \\
\hline 10386922 & 00289 & 12.747 & 12.540 & 11.534 & 11.220 & 11.187 & no & 0.92 & PHARO & $K$ & 0.2 & 1.0 & 3.1 & 5.0 & 6.2 & 6.5 \\
\hline 10933561 & 00291 & 12.848 & 12.642 & 11.680 & 11.399 & 11.320 & no & 0.69 & PHARO & $K$ & 0.3 & 1.0 & 3.0 & 4.5 & 5.0 & 5.1 \\
\hline 11547513 & 00295 & 12.324 & 12.155 & 11.260 & 10.984 & 10.951 & no & 0.77 & PHARO & $K$ & 0.9 & 1.8 & 3.6 & 5.7 & 6.7 & 6.9 \\
\hline 12785320 & 00298 & 12.713 & 12.355 & 11.295 & 10.946 & 10.885 & yes & $\ldots$ & PHARO & $J$ & 0.0 & 0.5 & 1.9 & 3.3 & 5.0 & 5.8 \\
\hline 12785320 & 00298 & 12.713 & 12.355 & 11.295 & 10.946 & 10.885 & yes & $\ldots$ & PHARO & $K$ & 0.5 & 1.2 & 3.1 & 4.5 & 4.9 & 5.8 \\
\hline 3642289 & 00301 & 12.730 & 12.586 & 11.722 & 11.508 & 11.456 & no & 0.72 & PHARO & $K$ & 0.0 & 0.9 & 3.0 & 4.7 & 5.3 & 5.4 \\
\hline 6029239 & 00304 & 12.549 & 12.377 & 11.472 & 11.192 & 11.109 & no & 0.83 & PHARO & $K$ & 0.7 & 1.7 & 4.2 & 5.6 & 6.5 & 6.7 \\
\hline 6289257 & 00307 & 12.797 & 12.650 & 11.806 & 11.552 & 11.488 & no & 0.73 & PHARO & $K$ & 0.0 & 0.9 & 3.1 & 4.7 & 5.2 & 5.3 \\
\hline 7050989 & 00312 & 12.459 & $\ldots$ & 10.804 & 10.573 & 10.519 & yes & $\ldots$ & NIRC2 & $K$ & 1.4 & 3.3 & 5.4 & 6.0 & 6.1 & 6.0 \\
\hline 7050989 & 00312 & 12.459 & $\ldots$ & 10.804 & 10.573 & 10.519 & yes & $\ldots$ & PHARO & $K$ & 0.2 & 1.3 & 3.2 & 5.5 & 7.1 & 7.7 \\
\hline 7419318 & 00313 & 12.990 & 12.736 & 11.650 & 11.229 & 11.165 & no & 0.81 & PHARO & $J$ & 0.4 & 1.3 & 2.8 & 4.8 & 7.1 & 8.1 \\
\hline 7419318 & 00313 & 12.990 & 12.736 & 11.650 & 11.229 & 11.165 & no & 0.81 & PHARO & $K$ & 0.5 & 1.8 & 3.7 & 5.3 & 6.9 & 7.1 \\
\hline 7603200 & 00314 & 12.925 & 12.457 & 10.293 & 9.680 & 9.506 & no & 0.91 & PHARO & $K$ & 0.3 & 1.1 & 3.0 & 4.9 & 6.3 & 6.6 \\
\hline 8008067 & 00316 & 12.701 & 12.494 & 11.530 & 11.222 & 11.167 & no & 0.82 & PHARO & $J$ & 0.0 & 0.5 & 1.6 & 3.2 & 5.4 & 6.9 \\
\hline 8008067 & 00316 & 12.701 & 12.494 & 11.530 & 11.222 & 11.167 & no & 0.82 & PHARO & $K$ & 0.3 & 1.4 & 3.2 & 4.9 & 6.1 & 6.4 \\
\hline 8753657 & 00321 & 12.520 & 12.312 & 11.340 & 11.035 & 10.970 & no & 0.92 & NIRC2 & $K$ & 2.8 & 4.3 & 6.1 & 6.7 & 6.8 & 6.8 \\
\hline 9880467 & 00326 & 12.960 & 12.960 & 14.774 & 13.236 & 13.085 & yes & $\ldots$ & PHARO & $K$ & 0.1 & 1.0 & 3.9 & 4.6 & 4.9 & 4.9 \\
\hline 9881662 & 00327 & 12.996 & 12.858 & 11.989 & 11.759 & 11.709 & no & 0.91 & PHARO & $K$ & 0.1 & 0.9 & 2.7 & 4.2 & 4.6 & 4.7 \\
\hline 10290666 & 00332 & 13.046 & 12.847 & 11.910 & 11.569 & 11.475 & no & 0.76 & PHARO & $K$ & 0.2 & 0.8 & 2.5 & 4.2 & 5.4 & 5.6 \\
\hline 10552611 & 00338 & 13.448 & 13.116 & 11.955 & 11.485 & 11.393 & no & 0.68 & PHARO & $K$ & 0.5 & 1.5 & 3.6 & 5.3 & 6.3 & 6.3 \\
\hline 10878263 & 00341 & 13.338 & 13.106 & 12.087 & 11.750 & 11.698 & no & 0.71 & ARIES & $K$ & 0.0 & 0.5 & 2.4 & 4.9 & 6.0 & 6.1 \\
\hline 10982872 & 00343 & 13.203 & 13.013 & 12.092 & 11.801 & 11.762 & no & 0.73 & PHARO & $K$ & 0.3 & 1.1 & 2.7 & 4.5 & 5.4 & 5.5 \\
\hline 11566064 & 00353 & 13.374 & 13.251 & 12.455 & 12.263 & 12.228 & yes & $\ldots$ & PHARO & $K$ & 0.1 & 0.9 & 2.4 & 3.6 & 4.6 & 4.8 \\
\hline 11568987 & 00354 & 13.235 & 13.057 & 12.063 & 11.775 & 11.708 & yes & $\ldots$ & PHARO & $K$ & 0.2 & 0.9 & 2.6 & 4.4 & 5.4 & 5.5 \\
\hline 7175184 & 00369 & 11.992 & 11.868 & 11.050 & 10.830 & 10.792 & no & 0.76 & PHARO & $K$ & 0.2 & 1.0 & 3.0 & 4.9 & 5.8 & 6.1 \\
\hline 12068975 & 00623 & 11.811 & 11.685 & 10.814 & 10.577 & 10.535 & no & 0.85 & NIRC2 & $K$ & 2.0 & 4.1 & 6.0 & 6.4 & 6.5 & 6.5 \\
\hline 4478168 & 00626 & 13.490 & 13.339 & 12.514 & 12.195 & 12.205 & yes & $\ldots$ & PHARO & $K$ & 0.8 & 1.8 & 4.5 & 5.5 & 5.8 & 6.0 \\
\hline
\end{tabular}


Table 1

(Continued)

\begin{tabular}{|c|c|c|c|c|c|c|c|c|c|c|c|c|c|c|c|c|}
\hline \multicolumn{7}{|c|}{ Kepler } & \multicolumn{4}{|c|}{ Observation } & \multicolumn{6}{|c|}{ Limiting Delta Magnitude $^{\mathrm{a}}$} \\
\hline KIC & KOI & $\begin{array}{l}\text { Kmag } \\
(\mathrm{mag})\end{array}$ & $\begin{array}{c}i \\
(\mathrm{mag})\end{array}$ & $\begin{array}{c}J \\
(\mathrm{mag})\end{array}$ & $\begin{array}{c}H \\
(\mathrm{mag})\end{array}$ & $\begin{array}{c}K \\
(\mathrm{mag})\end{array}$ & $\begin{array}{c}\text { Companion } \\
\text { within } 5^{\prime \prime}\end{array}$ & $\begin{array}{c}\text { Isolation } \\
\text { Probability }^{\mathrm{b}}\end{array}$ & Instrument & Filter & $\begin{array}{l}0.1 \\
(")\end{array}$ & $\begin{array}{l}0.2 \\
\left({ }^{\prime \prime}\right)\end{array}$ & $\begin{array}{l}0.5 \\
(")\end{array}$ & $\begin{array}{l}1.0 \\
(")\end{array}$ & $\begin{array}{l}2.0 \\
\left({ }^{\prime \prime}\right)\end{array}$ & $\begin{array}{l}4.0 \\
\left({ }^{\prime \prime}\right)\end{array}$ \\
\hline 4563268 & 00627 & 13.307 & 13.119 & 12.203 & 11.938 & 11.905 & no & 0.69 & PHARO & $K$ & 0.0 & 0.3 & 2.1 & 4.1 & 5.9 & $\overline{7.0}$ \\
\hline 5966154 & 00655 & 13.004 & 12.872 & 12.037 & 11.784 & 11.737 & no & 0.75 & PHARO & $K$ & 0.0 & 1.0 & 2.4 & 4.3 & 5.1 & 5.2 \\
\hline 6685609 & 00665 & 13.182 & 13.005 & 12.100 & 11.841 & 11.805 & no & 0.71 & PHARO & $K$ & 0.3 & 1.3 & 2.9 & 4.5 & 5.4 & 5.5 \\
\hline 7509886 & 00678 & 13.283 & 12.997 & 11.927 & 11.488 & 11.447 & no & 0.75 & PHARO & $K$ & 0.1 & 1.0 & 2.8 & 4.5 & 5.5 & 5.7 \\
\hline 7515212 & 00679 & 13.178 & 13.038 & 11.931 & 11.699 & 11.620 & no & 0.74 & PHARO & $K$ & 0.4 & 1.3 & 3.0 & 4.7 & 5.7 & 5.9 \\
\hline 9590976 & 00710 & 13.294 & 13.128 & 12.319 & 12.176 & 12.103 & no & 0.68 & PHARO & $K$ & 0.0 & 1.0 & 2.6 & 4.2 & 5.0 & 5.1 \\
\hline 9873254 & 00717 & 13.387 & 13.182 & 12.194 & 11.868 & 11.793 & no & 0.72 & PHARO & $K$ & 0.2 & 1.3 & 3.2 & 4.7 & 5.6 & 5.8 \\
\hline 9950612 & 00719 & 13.177 & 12.899 & 11.206 & 10.672 & 10.550 & no & 0.94 & NIRC2 & $K$ & 2.7 & 4.5 & 6.6 & 7.8 & 8.0 & 8.0 \\
\hline 11013201 & 00972 & 9.275 & 9.392 & 8.816 & 8.765 & 8.736 & no & 0.86 & PHARO & $K$ & 1.3 & 2.5 & 5.1 & 6.8 & 8.8 & 9.3 \\
\hline 1871056 & 01001 & 13.038 & 12.851 & 11.918 & 11.692 & 11.591 & yes & $\ldots$ & PHARO & $K$ & 0.2 & 1.0 & 2.7 & 4.4 & 5.4 & 5.6 \\
\hline 8280511 & 01151 & 13.404 & 13.198 & 12.198 & 11.819 & 11.745 & yes & $\ldots$ & PHARO & $K$ & 0.3 & 1.3 & 3.1 & 4.6 & 5.7 & 5.8 \\
\hline 10350571 & 01175 & 13.290 & 13.075 & 12.061 & 11.704 & 11.617 & no & 0.67 & PHARO & $K$ & 0.0 & 0.8 & 2.4 & 4.1 & 5.2 & 5.4 \\
\hline 3939150 & 01215 & 13.420 & 13.226 & 12.288 & 12.003 & 11.966 & no & 0.68 & PHARO & $K$ & 0.5 & 1.4 & 3.5 & 5.1 & 5.7 & 5.8 \\
\hline 6448890 & 01241 & 12.440 & 12.090 & 10.813 & 10.330 & 10.227 & no & 0.81 & NIRC2 & $K$ & 2.1 & 3.6 & 5.4 & 6.0 & 6.1 & 6.0 \\
\hline 6448890 & 01241 & 12.440 & 12.090 & 10.813 & 10.330 & 10.227 & no & 0.81 & PHARO & $K$ & 0.1 & 0.9 & 2.8 & 5.0 & 6.8 & 7.5 \\
\hline 10794087 & 01316 & 11.926 & 11.694 & 10.894 & 10.606 & 10.562 & yes & $\ldots$ & ARIES & $K$ & 0.4 & 1.6 & 3.5 & 5.8 & 7.9 & 8.2 \\
\hline 10794087 & 01316 & 11.926 & 11.694 & 10.894 & 10.606 & 10.562 & yes & $\ldots$ & NIRC2 & $K$ & 2.4 & 4.4 & 6.9 & 7.6 & 7.7 & 7.7 \\
\hline 11336883 & 01445 & 12.320 & 12.209 & 11.406 & 11.171 & 11.151 & no & 0.86 & PHARO & $K$ & 0.2 & 1.0 & 2.8 & 4.9 & 6.4 & 6.9 \\
\hline 7869917 & 01525 & 12.082 & 12.009 & 11.250 & 11.065 & 11.039 & no & 0.71 & PHARO & $K$ & 0.4 & 1.1 & 3.0 & 4.9 & 6.5 & 7.1 \\
\hline 4741126 & 01534 & 13.470 & 13.325 & 12.539 & 12.270 & 12.241 & no & 0.69 & PHARO & $K$ & 0.4 & 1.1 & 3.0 & 4.5 & 5.1 & 5.2 \\
\hline 6268648 & 01613 & 11.049 & $\ldots$ & 10.588 & 10.316 & 10.282 & yes & $\ldots$ & NIRC2 & $K$ & 2.4 & 4.5 & 6.3 & 7.6 & 7.7 & 7.7 \\
\hline 6268648 & 01613 & 11.049 & $\ldots$ & 10.588 & 10.316 & 10.282 & yes & $\ldots$ & PHARO & $K$ & 0.0 & 0.4 & 4.4 & 5.9 & 7.0 & 7.3 \\
\hline 6975129 & 01628 & 12.949 & 12.775 & 11.902 & 11.664 & 11.596 & no & 0.83 & PHARO & $K$ & 0.2 & 1.0 & 2.9 & 4.5 & 4.9 & 5.0 \\
\hline 6616218 & 01692 & 12.557 & 12.313 & 11.242 & 10.850 & 10.778 & yes & & PHARO & $K$ & 0.2 & 1.0 & 4.1 & 5.3 & 6.4 & 6.8 \\
\hline 9909735 & 01779 & 13.297 & 13.077 & 12.148 & 11.832 & 11.766 & no & 0.80 & NIRC2 & $K$ & 1.8 & 4.1 & 5.2 & 5.5 & 5.5 & 5.4 \\
\hline 9909735 & 01779 & 13.297 & 13.077 & 12.148 & 11.832 & 11.766 & no & 0.80 & PHARO & $K$ & 0.1 & 1.0 & 2.7 & 4.5 & 5.5 & 5.6 \\
\hline 11551692 & 01781 & 12.231 & 11.884 & 10.641 & 10.161 & 10.062 & yes & $\ldots$ & NIRC2 & $J$ & 1.7 & 2.6 & 4.2 & 5.5 & 5.8 & 5.6 \\
\hline 11551692 & 01781 & 12.231 & 11.884 & 10.641 & 10.161 & 10.062 & yes & $\ldots$ & NIRC2 & $K$ & 1.5 & 3.3 & 5.1 & 5.8 & 5.9 & 5.8 \\
\hline 11551692 & 01781 & 12.231 & 11.884 & 10.641 & 10.161 & 10.062 & yes & $\ldots$ & PHARO & $K$ & 0.1 & 1.1 & 3.0 & 5.2 & 6.8 & 7.3 \\
\hline 9529744 & 01806 & 13.474 & 13.337 & 12.546 & 12.283 & 12.307 & yes & $\ldots$ & PHARO & $K$ & 0.3 & 1.2 & 3.1 & 4.5 & 5.0 & 4.9 \\
\hline 8240797 & 01809 & 12.706 & 12.474 & 11.621 & 11.300 & 11.249 & no & 0.73 & PHARO & $K$ & 0.2 & 1.0 & 3.1 & 5.3 & 6.1 & 6.2 \\
\hline 2989404 & 01824 & 12.722 & 12.567 & 11.689 & 11.423 & 11.354 & no & 0.73 & PHARO & $K$ & 0.3 & 1.3 & 3.3 & 5.0 & 5.9 & 6.0 \\
\hline 10130039 & 01909 & 12.776 & 12.612 & 11.710 & 11.448 & 11.409 & no & 0.73 & PHARO & $K$ & 0.3 & 1.2 & 3.2 & 4.8 & 5.6 & 5.7 \\
\hline 10136549 & 01929 & 12.727 & 12.530 & 11.537 & 11.257 & 11.183 & yes & $\ldots$ & PHARO & $K$ & 0.3 & 1.1 & 3.2 & 4.9 & 5.7 & 5.8 \\
\hline 5511081 & 01930 & 12.119 & 11.957 & 11.098 & 10.841 & 10.756 & no & 0.85 & NIRC2 & $K$ & 2.6 & 4.6 & 6.7 & 7.3 & 7.4 & 7.4 \\
\hline 5202905 & 01932 & 12.345 & 12.366 & 11.725 & 11.629 & 11.583 & yes & $\ldots$ & NIRC2 & $H$ & 1.4 & 2.8 & 4.4 & 5.1 & 5.3 & 5.3 \\
\hline 5202905 & 01932 & 12.345 & 12.366 & 11.725 & 11.629 & 11.583 & yes & $\ldots$ & NIRC2 & $J$ & 1.3 & 2.4 & 3.9 & 5.0 & 5.3 & 5.2 \\
\hline 5202905 & 01932 & 12.345 & 12.366 & 11.725 & 11.629 & 11.583 & yes & $\ldots$ & NIRC2 & $K$ & 1.5 & 3.8 & 4.9 & 5.4 & 5.4 & 5.3 \\
\hline 5202905 & 01932 & 12.345 & 12.366 & 11.725 & 11.629 & 11.583 & yes & $\ldots$ & PHARO & $K$ & 0.2 & 1.1 & 3.1 & 5.3 & 6.6 & 6.8 \\
\hline 9892816 & 01955 & 13.147 & 13.025 & 12.220 & 11.999 & 11.957 & no & 0.76 & PHARO & $K$ & 0.3 & 1.1 & 3.0 & 4.6 & 5.3 & 5.4 \\
\hline 12154526 & 02004 & 13.351 & 13.150 & 12.174 & 11.872 & 11.803 & no & 0.78 & PHARO & $K$ & 0.4 & 1.2 & 2.8 & 4.5 & 5.5 & 5.7 \\
\hline 5384079 & 02011 & 12.556 & 12.419 & 11.708 & 11.454 & 11.377 & yes & $\ldots$ & PHARO & K & 0.1 & 0.9 & 2.7 & 4.8 & 6.2 & 6.5 \\
\hline 9489524 & 02029 & 12.957 & 12.694 & 11.610 & 11.178 & 11.132 & no & 0.91 & NIRC2 & $K$ & 2.2 & 4.4 & 6.4 & 7.3 & 7.3 & 7.3 \\
\hline 2307415 & 02053 & 12.992 & 12.839 & 12.000 & 11.745 & 11.704 & no & 0.71 & PHARO & K & 0.1 & 0.8 & 2.5 & 4.2 & 4.7 & 4.7 \\
\hline 12301181 & 02059 & 12.906 & 12.558 & 11.305 & 10.791 & 10.664 & yes & $\ldots$ & NIRC2 & $K$ & 2.4 & 4.0 & 5.6 & 7.4 & 7.8 & 7.8 \\
\hline
\end{tabular}




\begin{tabular}{|c|c|c|c|c|c|c|c|c|c|c|c|c|c|c|c|c|}
\hline \multicolumn{7}{|c|}{ Kepler } & \multicolumn{4}{|c|}{ Observation } & \multicolumn{6}{|c|}{ Limiting Delta Magnitude $^{\mathrm{a}}$} \\
\hline KIC & KOI & $\begin{array}{l}\text { Kmag } \\
(\mathrm{mag})\end{array}$ & $\begin{array}{c}i \\
(\mathrm{mag})\end{array}$ & $\begin{array}{c}J \\
(\mathrm{mag})\end{array}$ & $\begin{array}{c}H \\
(\mathrm{mag})\end{array}$ & $\begin{array}{c}K \\
(\mathrm{mag})\end{array}$ & $\begin{array}{c}\text { Companion } \\
\text { within } 5^{\prime \prime}\end{array}$ & $\begin{array}{c}\text { Isolation } \\
\text { Probability }\end{array}$ & Instrument & Filter & $\begin{array}{l}0.1 \\
(\prime)\end{array}$ & $\begin{array}{l}0.2 \\
\left({ }^{\prime \prime}\right)\end{array}$ & $\begin{array}{l}0.5 \\
(")\end{array}$ & $\begin{array}{l}1.0 \\
(")\end{array}$ & $\begin{array}{l}2.0 \\
(")\end{array}$ & $\begin{array}{l}4.0 \\
(\prime)\end{array}$ \\
\hline 12301181 & 02059 & 12.906 & 12.558 & 11.305 & 10.791 & 10.664 & yes & & PHARO & $K$ & 0.0 & 0.0 & 1.7 & 3.6 & 4.8 & 4.9 \\
\hline 6021193 & 02148 & 13.353 & 13.112 & 12.111 & 11.755 & 11.697 & no & 0.68 & PHARO & K & 0.5 & 1.5 & 3.6 & 5.2 & 6.0 & 6.2 \\
\hline 9006186 & 02169 & 12.404 & 12.172 & 11.137 & 10.735 & 10.662 & yes & & PHARO & $K$ & 0.2 & 1.1 & 2.9 & 5.0 & 6.8 & 7.1 \\
\hline 11774991 & 02173 & 12.879 & 12.522 & 11.243 & 10.752 & 10.674 & no & 0.94 & NIRC2 & $K$ & 2.8 & 4.6 & 6.6 & 7.3 & 7.5 & 7.4 \\
\hline 9022166 & 02175 & 12.848 & 12.626 & 11.600 & 11.229 & 11.175 & no & 0.85 & NIRC2 & $K$ & 2.9 & 4.6 & 6.5 & 7.1 & 7.2 & 7.2 \\
\hline 3867615 & 02289 & 13.358 & 13.193 & 12.341 & 12.092 & 12.005 & yes & $\ldots$ & PHARO & $K$ & 0.3 & 1.3 & 3.1 & 4.6 & 5.6 & 5.7 \\
\hline 8013439 & 02352 & 10.421 & $\ldots$ & 9.721 & 9.547 & 9.504 & no & 0.92 & $\mathrm{NIRC} 2$ & $K$ & 2.5 & 4.6 & 6.8 & 7.5 & 7.5 & 7.5 \\
\hline 8013439 & 02352 & 10.421 & $\ldots$ & 9.721 & 9.547 & 9.504 & no & 0.92 & PHARO & $K$ & 1.1 & 2.7 & 5.0 & 6.8 & 7.8 & 8.1 \\
\hline 12306058 & 02541 & 13.007 & 12.717 & 11.564 & 11.072 & 10.970 & no & 0.66 & PHARO & $K$ & 0.2 & 0.9 & 2.7 & 4.6 & 5.8 & 6.1 \\
\hline 8883329 & 02595 & 13.223 & 13.107 & 12.325 & 12.087 & 11.995 & no & 0.68 & PHARO & $K$ & 0.2 & 1.0 & 2.6 & 4.4 & 5.5 & 5.7 \\
\hline 11253827 & 02672 & 11.921 & 11.703 & 10.672 & 10.356 & 10.285 & yes & & PHARO & $K$ & 0.2 & 1.4 & 4.0 & 5.9 & 6.8 & 6.8 \\
\hline 8022489 & 02674 & 13.349 & 13.159 & 12.169 & 11.859 & 11.825 & no & 0.81 & PHARO & $K$ & 0.5 & 1.5 & 3.1 & 4.8 & 5.6 & 5.7 \\
\hline 7202957 & 02687 & 10.158 & 9.973 & 9.052 & 8.761 & 8.693 & no & 0.88 & PHARO & $K$ & 0.9 & 2.9 & 5.6 & 7.5 & 8.8 & 8.9 \\
\hline 11071200 & 02696 & 12.998 & 12.901 & 12.188 & 12.032 & 11.950 & no & 0.66 & PHARO & $K$ & 0.0 & 0.7 & 2.2 & 4.0 & 4.7 & 4.8 \\
\hline 12206313 & 02714 & 13.312 & 13.160 & 12.277 & 12.065 & 11.987 & no & 0.71 & PHARO & $K$ & 0.3 & 1.3 & 3.1 & 4.7 & 5.4 & 5.5 \\
\hline 6026737 & 02949 & 13.313 & 13.135 & 12.222 & 11.973 & 11.903 & yes & $\ldots$ & PHARO & $K$ & 0.5 & 1.6 & 3.4 & 5.2 & 5.8 & 5.9 \\
\hline 6278762 & 03158 & 8.717 & $\ldots$ & 7.244 & 6.772 & 6.703 & yes & $\ldots$ & NIRC2 & $K$ & 2.7 & 4.6 & 5.8 & 6.1 & 6.1 & 6.1 \\
\hline 6278762 & 03158 & 8.717 & $\ldots$ & 7.244 & 6.772 & 6.703 & yes & $\ldots$ & PHARO & $J$ & 0.0 & 1.6 & 3.5 & 5.3 & 7.1 & 8.9 \\
\hline 6278762 & 03158 & 8.717 & $\ldots$ & 7.244 & 6.772 & 6.703 & yes & $\ldots$ & PHARO & $K$ & 1.5 & 3.4 & 5.8 & 7.4 & 8.9 & 9.7 \\
\hline 9002538 & 03196 & 11.525 & 11.405 & 10.547 & 10.335 & 10.276 & no & 0.90 & NIRC2 & $K$ & 2.6 & 4.4 & 6.6 & 7.5 & 7.6 & 7.6 \\
\hline 9002538 & 03196 & 11.525 & 11.405 & 10.547 & 10.335 & 10.276 & no & 0.90 & PHARO & $K$ & 0.3 & 1.3 & 3.9 & 5.5 & 6.6 & 7.0 \\
\hline 8644365 & 03384 & 13.204 & 13.008 & 12.022 & 11.757 & 11.724 & no & 0.72 & PHARO & $K$ & 0.4 & 1.4 & 3.1 & 4.8 & 5.7 & 5.8 \\
\hline 3561464 & 03398 & 13.489 & 13.361 & 12.556 & 12.311 & 12.289 & no & 0.80 & PHARO & $K$ & 0.9 & 2.4 & 4.9 & 5.5 & 5.9 & 5.9 \\
\hline 11754430 & 03403 & 13.102 & 12.921 & 12.012 & 11.694 & 11.638 & no & 0.79 & PHARO & $K$ & 0.2 & 1.1 & 2.9 & 4.7 & 5.6 & 5.7 \\
\hline 9117416 & 03425 & 13.266 & 12.957 & 11.897 & 11.610 & 11.514 & no & 0.84 & NIRC2 & $K$ & 2.3 & 4.3 & 6.4 & 7.2 & 7.3 & 7.3 \\
\hline 6058816 & 03500 & 13.214 & 13.038 & 12.161 & 11.870 & 11.826 & yes & $\ldots$ & PHARO & $K$ & 0.3 & 1.1 & 2.9 & 4.4 & 5.2 & 5.4 \\
\hline 2581316 & 03681 & 11.690 & $\ldots$ & 10.953 & 10.728 & 10.688 & no & 0.92 & NIRC2 & K & 2.2 & 4.6 & 5.8 & 5.9 & 5.9 & 5.9 \\
\hline 4164922 & 03864 & 12.914 & 12.604 & 11.489 & 11.013 & 10.915 & no & 0.77 & PHARO & $K$ & 0.1 & 1.0 & 2.9 & 4.7 & 5.5 & 5.6 \\
\hline 11967788 & 04021 & 13.166 & 12.513 & 11.797 & 11.538 & 11.487 & yes & $\ldots$ & PHARO & $K$ & 0.0 & 0.4 & 1.8 & 2.9 & 4.0 & 4.4 \\
\hline 7100673 & 04032 & 12.639 & 12.432 & 11.421 & 11.034 & 10.989 & no & 0.77 & PHARO & $K$ & 0.0 & 0.6 & 1.9 & 4.1 & 6.0 & 6.4 \\
\hline 5688683 & 04097 & 13.435 & 12.965 & 11.614 & 10.958 & 10.841 & no & 0.85 & PHARO & $K$ & 0.3 & 1.4 & 3.3 & 5.1 & 6.2 & 6.5 \\
\hline 8890924 & 04269 & 13.263 & 12.943 & 11.718 & 11.249 & 11.136 & no & 0.79 & PHARO & $K$ & 0.0 & 0.9 & 2.5 & 4.4 & 5.7 & 6.0 \\
\hline 4548011 & 04288 & 12.400 & 12.246 & 11.331 & 11.106 & 11.025 & yes & $\ldots$ & PHARO & $K$ & 0.3 & 1.1 & 3.0 & 5.3 & 6.7 & 7.1 \\
\hline
\end{tabular}

Notes.

${ }^{\mathrm{a}}$ Limiting Delta Magnitudes are the $5 \sigma$ limit.

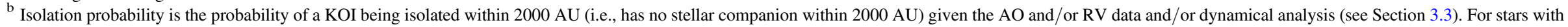
detected nearby stellar companions, the physical association probability can be found in Table 2 . 
Table 2

Visual Companion Detections with AO Data for Kepler MTPS

\begin{tabular}{|c|c|c|c|c|c|c|c|c|c|c|c|}
\hline \multirow[t]{2}{*}{ KOI } & \multirow[t]{2}{*}{ Star\# } & \multirow[t]{2}{*}{ Telescope } & \multirow[t]{2}{*}{ Filter } & \multirow{2}{*}{$\begin{array}{c}\Delta \mathrm{Mag}^{\mathrm{a}} \\
(\mathrm{mag})\end{array}$} & \multicolumn{2}{|c|}{ Separation $^{\mathrm{b}}$} & \multicolumn{2}{|c|}{ Distance $^{c}$} & \multirow{2}{*}{$\begin{array}{c}\text { PA } \\
(\mathrm{deg})\end{array}$} & \multirow{2}{*}{$\begin{array}{l}\text { Association }^{\mathrm{d}} \\
\text { Probability }\end{array}$} & \multirow[t]{2}{*}{ Ref. $^{\mathrm{e}}$} \\
\hline & & & & & $(\operatorname{arcsec})$ & $(\mathrm{AU})$ & $\begin{array}{l}\text { Primary } \\
(\mathrm{pc})\end{array}$ & $\begin{array}{l}\text { Secondary } \\
\text { (pc) }\end{array}$ & & & \\
\hline K00005 & 1 & Keck & $\mathrm{K}$ & 2.20 & 0.14 & 40.12 & $286.6_{-15.8}^{71.1}$ & $\ldots$ & 307.4 & $>0.90$ & CFOP \\
\hline K00070 & 1 & Palomar & $\mathrm{J}$ & 4.41 & 3.77 & 1052.60 & $279.5_{-23.6}^{25.3}$ & $\ldots$ & 51.8 & 0.52 & A12 \\
\hline K00102 & 1 & Palomar & $\mathrm{J}$ & 1.12 & 2.84 & 934.31 & $329.4_{-30.5}^{75.0}$ & $\ldots$ & 222.2 & $>0.90$ & A12 \\
\hline K00108 & 1 & Palomar & $\mathrm{J}$ & 5.71 & 2.51 & 891.07 & $354.6_{-39.2}^{45.4}$ & $\ldots$ & 285.2 & 0.48 & A12 \\
\hline K00108 & 2 & Palomar & $\mathrm{J}$ & 5.60 & 3.23 & 1145.12 & $354.6_{-39.2}^{45.4}$ & $\ldots$ & 100.8 & 0.30 & A12 \\
\hline K00108 & 3 & Palomar & $\mathrm{J}$ & 6.60 & 5.00 & 1773.09 & $354.6_{-39.2}^{45.4}$ & $\ldots$ & 112.5 & 0.00 & A12 \\
\hline K00115 & 1 & MMT & K & 5.06 & 4.00 & 2168.27 & $542.1_{-97.0}^{140.6}$ & $\ldots$ & 89.7 & 0.33 & A12 \\
\hline K00119 & 1 & Palomar & $\mathrm{J}$ & 0.16 & 1.05 & 327.89 & $313.0_{-62.2}^{106.8}$ & $380.8_{-154.5}^{499.6}$ & 119.1 & $>0.90$ & this work \\
\hline K00119 & 1 & Palomar & K & 0.22 & 1.04 & 326.17 & $313.0_{-62.2}^{106.2}$ & $380.8_{-154.5}^{499.6}$ & 120.2 & $>0.90$ & this work \\
\hline K00148 & 1 & Palomar & $\mathrm{J}$ & 4.75 & 2.51 & 775.44 & $308.7_{-17.2}^{27.0}$ & $\ldots$ & 245.6 & 0.78 & A12 \\
\hline K00148 & 2 & Palomar & $\mathrm{J}$ & 3.14 & 4.43 & 1368.74 & $308.7_{-17.2}^{27.0}$ & $\ldots$ & 220.4 & 0.73 & A12 \\
\hline K00279 & 1 & Keck & $\mathrm{K}$ & 2.35 & 0.92 & 247.44 & $268.6_{-46.3}^{187.6}$ & $\ldots$ & 247.3 & $>0.90$ & CFOP \\
\hline K00282 & 1 & Palomar & $\mathrm{K}$ & 3.86 & 4.16 & 1408.24 & $338.8_{-26.5}^{16.9}$ & $\ldots$ & 210.3 & 0.84 & CFOP \\
\hline K00284 & 1 & Palomar & $\mathrm{J}$ & 0.24 & 0.87 & 229.45 & $264.7_{-39.4}^{34.4}$ & $339.5_{-146.8}^{347.4}$ & 95.8 & $>0.90$ & A12 \\
\hline K00284 & 1 & Palomar & $\mathrm{K}$ & 0.24 & 0.86 & 226.48 & $264.7_{-39.4}^{34.4}$ & $339.5_{-146.8}^{344.4}$ & 96.7 & $>0.90$ & A12 \\
\hline K00285 & 1 & Palomar & $\mathrm{J}$ & 4.19 & 1.50 & 676.86 & $452.7_{-47.0}^{18.4}$ & $3855.9_{-3163.9}^{2632.5}$ & 137.7 & $>0.90$ & CFOP \\
\hline K00285 & 1 & Palomar & $\mathrm{K}$ & 4.08 & 1.50 & 677.09 & $452.7_{-47.0}^{18.4}$ & $3855.9_{-3163.9}^{2632.5}$ & 137.7 & $>0.90$ & CFOP \\
\hline K00298 & 1 & Palomar & $\mathrm{J}$ & 0.24 & 2.00 & 581.07 & $290.2_{-54.4}^{300.0}$ & $247.2_{-68.1}^{335.0}$ & 272.8 & $>0.90$ & this work \\
\hline K00298 & 1 & Palomar & $\mathrm{K}$ & 0.08 & 1.96 & 570.05 & $290.2_{-54.4}^{300.0}$ & $247.2_{-68.1}^{335.0}$ & 272.5 & $>0.90$ & this work \\
\hline K00312 & 1 & Palomar & $\mathrm{K}$ & 6.67 & 3.01 & 950.62 & $316.1_{-25.9}^{33.3}$ & $\ldots$ & 104.4 & 0.34 & this work \\
\hline K00312 & 2 & Palomar & $\mathrm{K}$ & 5.84 & 4.97 & 1569.91 & $316.1_{-25.9}^{33.3}$ & $\ldots$ & 121.7 & 0.33 & this work \\
\hline K00326 & 1 & Palomar & $\mathrm{K}$ & 1.03 & 3.49 & 27865.11 & $7989.4_{-1200.3}^{1953.2}$ & $\ldots$ & 269.4 & 0.89 & this work \\
\hline K00353 & 1 & Palomar & $\mathrm{K}$ & 3.07 & 1.04 & 820.45 & $789.7_{-103.2}^{151.9}$ & $\ldots$ & 23.0 & $>0.90$ & this work \\
\hline K00353 & 2 & Palomar & $\mathrm{K}$ & 4.15 & 1.43 & 1131.97 & $789.7_{-103.2}^{151.9}$ & $\ldots$ & 236.3 & $>0.90$ & this work \\
\hline K00354 & 1 & Palomar & K & 4.83 & 3.73 & 1425.50 & $382.1_{-25.5}^{29.8}$ & $\ldots$ & 210.1 & 0.36 & this work \\
\hline K00626 & 1 & Palomar & $\mathrm{K}$ & 5.30 & 2.75 & 1463.00 & $532.3_{-43.4}^{39.1}$ & $\cdots$ & 167.9 & 0.21 & this work \\
\hline K01151 & 1 & Palomar & $\mathrm{K}$ & 2.25 & 0.76 & 316.71 & $419.5_{-50.0}^{53.7}$ & $\ldots$ & 306.6 & $>0.90$ & this work \\
\hline K01316 & 1 & MMT & K & 5.81 & 2.78 & 1249.69 & $449.6_{-96.3}^{185.2}$ & $\ldots$ & 4.8 & 0.68 & CFOP (Dupree) \\
\hline K01613 & 1 & Keck & $\mathrm{K}$ & 1.00 & 0.22 & 79.49 & $364.3_{-19.1}^{21.7}$ & $\ldots$ & 184.6 & $>0.90$ & CFOP \\
\hline K01613 & 1 & Palomar & $\mathrm{K}$ & 1.16 & 0.21 & 75.31 & $364.3_{-19.1}^{21.7}$ & $\ldots$ & 183.4 & $>0.90$ & CFOP \\
\hline K01692 & 1 & Palomar & $\mathrm{K}$ & 6.36 & 3.17 & 841.66 & $265.4_{-19.8}^{14.6}$ & $\ldots$ & 337.2 & 0.31 & this work \\
\hline K01781 & 1 & Keck & $\mathrm{J}$ & 2.71 & 3.48 & 607.66 & $174.8_{-14.8}^{10.7}$ & $508.7_{-178.8}^{569.0}$ & 332.4 & $>0.90$ & this work \\
\hline K01781 & 1 & Keck & K & 2.35 & 3.47 & 606.92 & $174.8_{-14.8}^{10.7}$ & $508.7_{-178.8}^{569.0}$ & 332.2 & $>0.90$ & this work \\
\hline K01781 & 1 & Palomar & $\mathrm{K}$ & 2.29 & 3.43 & 599.24 & $174.8_{-14.8}^{10.7}$ & $508.7_{-178.8}^{569.0}$ & 332.4 & $>0.90$ & this work \\
\hline K01806 & 1 & Palomar & $\mathrm{K}$ & 1.45 & 3.43 & 2096.38 & $612.1_{-70.2}^{62.3}$ & $\ldots$ & 249.7 & 0.90 & this work \\
\hline K01929 & 1 & Palomar & K & 4.86 & 1.37 & 835.32 & $608.8_{-162.1}^{64.2}$ & $\ldots$ & 163.0 & $>0.90$ & this work \\
\hline
\end{tabular}


Table 2

(Continued)

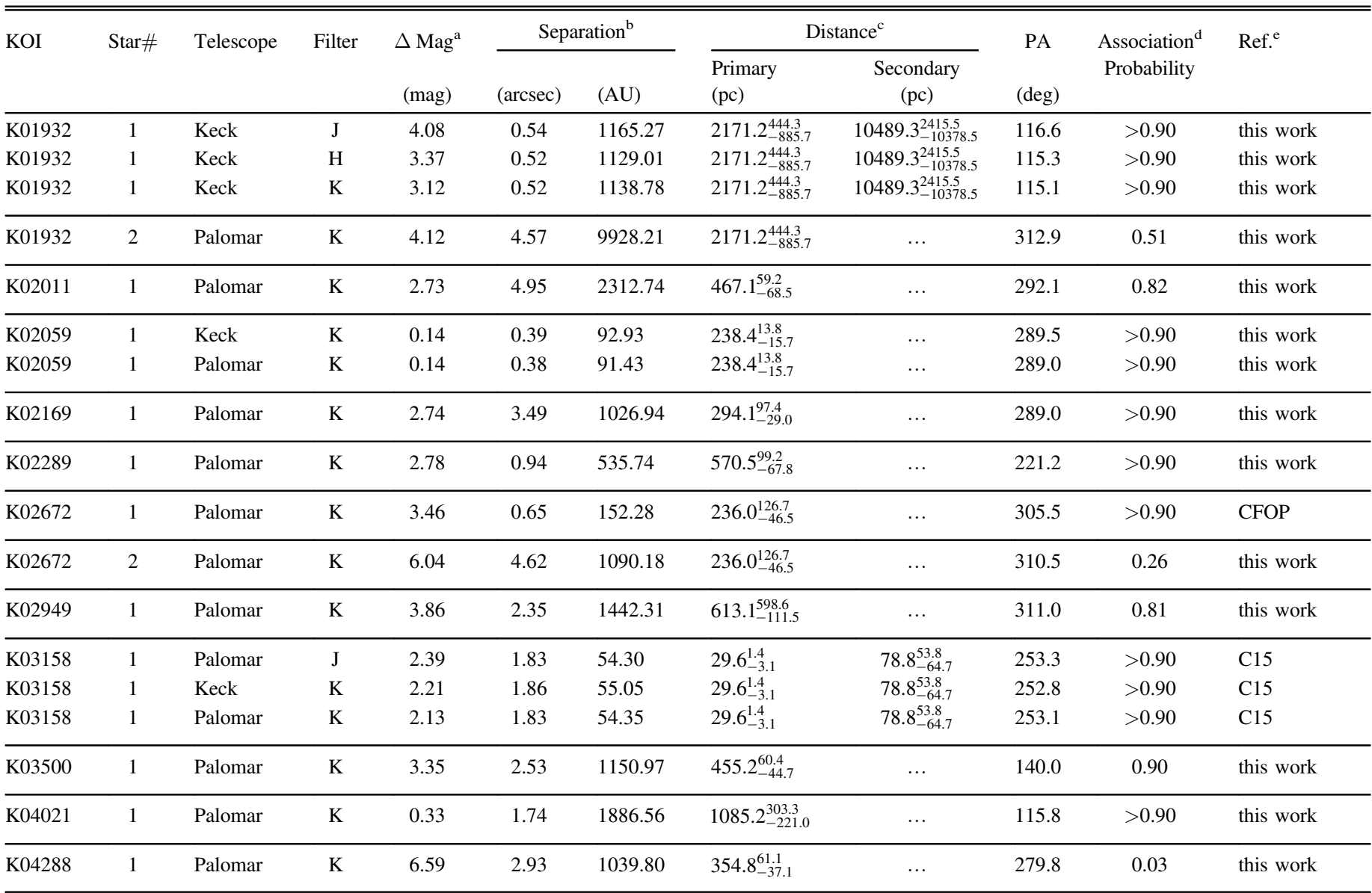

Notes.

a Typical $\Delta$ Mag uncertainty is $0.1 \mathrm{mag}$. The uncertainty is estimated from the companion injection simulation described in Section 3.3 .

b Typical angular separation uncertainty is $0 .{ }^{\prime \prime} 05$. The uncertainty is estimated from the companion injection simulation described in Section 3.3 .


more details).

d Association probability has $10 \%$ uncertainty due to statistical error in simulation.

e AO images from CFOP are provided by David Ciardi unless otherwise noted.

References. A12-Adams et al. (2012); C15-Campante et al. (2015).

Kepler objects of interest (KOIs) that satisfy the following criteria: (1) disposition of either Candidate or Confirmed; (2) with at least two planet candidates; (3) Kepler magnitude $\left(K_{P}\right)$ brighter than 13.5. The above selection criteria resulted in 138 MTPSs in Wang et al. (2014b). With the updated Exoplanet Archive, the selection criteria resulted in 208 MTPSs. In this paper, we focus on the 138 MTPSs to be consistent with previous work. Their stellar and orbital parameters can be found in Tables 2 and 3 in Wang et al. (2014b).

Most MTPSs in our sample are true planetary systems based on a statistical analysis by Lissauer et al. (2012). Subsequent papers on Kepler MTPS validated 851 planet candidates in 340 systems (Lissauer et al. 2014; Rowe et al. 2014), 66 MTPSs in our sample are included in those validated systems. Furthermore, 25 additional MTPSs in our sample are confirmed planetary systems, and the remaining 47 MTPSs have the disposition of a planet candidate according to the latest NASA Exoplanet Archive. Therefore, the false-positive rate for the MTPS sample studied in this paper should be extremely low.

\subsection{AO Data Acquisition}

\subsubsection{Archival AO Data for Follow-up Observations}

We checked the continually updated CFOP. To avoid repeated AO observations, we only observed KOIs that did not received AO follow-up observations. Some of the KOIs without AO data may have speckle imaging (e.g., Horch et al. 2012, 2014) or lucky imaging data (e.g., Lillo-Box et al. 2012, 2014), but we re-observed these KOIs at Palomar and Keck Observatory because near-infrared AO images provide deeper sensitivity and/or higher spatial resolution. For the same reason, we re-observed KOIs that have been observed by the Robo-AO project (Law et al. 2014). For those KOIs whose AO data from the Palomar, MMT, or Keck telescope were available through CFOP, we used the archival AO data. In total, AO data 
for 65 KOIs were obtained from CFOP, and AO data for 73 KOIs were obtained by new observations at Palomar and Keck observatory.

\subsubsection{AO Imaging with PHARO at Palomar}

We observed 68 KOIs in the sample with the PHARO instrument (Brandl et al. 1997; Hayward et al. 2001) at the Palomar 200 inch telescope (San Diego County, CA). The observations were made between UT July 13 and 17 in 2014 with seeing varying between 1 ". 0 and 2". 5 . PHARO is behind the Palomar-3000 AO system, which provides an on-sky Strehl of $86 \%$ in the $K$ band (Burruss 2014). The pixel scale of PHARO is 25 mas pixel $^{-1}$. With a mosaic $1 \mathrm{~K} \times 1 \mathrm{~K}$ detector, the field of view (FOV) is $25^{\prime \prime} \times 25^{\prime \prime}$. We normally obtained the first image in the $K$ band with a five-point dither pattern, which had a throw of 2"!5. AO images in the $K$ band provide higher sensitivity to bound companions with late spectral type than $J$ - and $H$-band images. Furthermore, the AO correction in the $K$ band is better and offers a better characterized pointspread function (PSF). This is because image quality improves toward longer wavelengths for a given wavefront sensing and correcting error (Davies \& Kasper 2012). A better image with a more stable PSF facilitates companion detection and characterization. Exposure time was set such that the peak flux of the $\mathrm{KOI}$ is at least 10,000 ADU for each frame, which is within the linear range of the detector. If a stellar companion was detected, we observed the KOI in the $J$ and $H$ bands right after the $K$-band observation. The color information is useful for estimating the stellar properties of the stellar companion and determining whether the companion is physically bound (see Section 3.2). Nearly simultaneous $J-, H$,, and $K$-band observations help to minimize the influence of any time variability of the target.

\subsubsection{AO Imaging with NIRC2 at Keck II}

We observed five KOIs in the sample with the NIRC2 instrument (Wizinowich et al. 2000) at the Keck II telescope (Mauna Kea, HI). The observations were made on UT July 18 and August 18 in 2014 with excellent/good seeing between 0 ". 3 and $0 . " 8$. NIRC2 is a near-infrared imager designed for the Keck AO system. We selected the narrow camera mode, which has a pixel scale of 10 mas pixel ${ }^{-1}$. The FOV is thus $10^{\prime \prime} \times 10^{\prime \prime}$ for a mosaic $1 \mathrm{~K} \times 1 \mathrm{~K}$ detector. We started the observation in the $K$ band for each KOI for the same reason stated in Section 2.2.2 and followed by $J$ - and $H$-band observations if any stellar companions were found. The exposure time setting is the same as the PHARO observation: we ensured that the peak flux is at least 10,000 ADU for each frame. We used a three-point dither pattern with a throw of 2 " 5 . We avoided the lower left quadrant in the dither pattern because it has a much higher instrumental noise than the other three quadrants on the detector.

\section{DATA ANALYSES}

\subsection{Contrast Curve and Detections}

The raw data were processed using standard techniques to replace bad pixels, subtract dark, flat-field, subtract sky background, align, and co-add frames. We constructed a bad pixel map using dark frames. Pixels with dark currents that deviated more than $5 \sigma$ from their surrounding pixels were recorded as bad pixels. Their values were replaced with the median flux of surrounding pixels. Dark frames were obtained with the exact same setting as the science frames, e.g., exposure time, co-adds, and readout mode. After dark subtraction, each science frame was corrected for flat fielding. The dithered science frames provided an estimate of the sky background that was subtracted off from the science frames. The darksubtracted, flat-fielded, sky-removed science frames were then co-added, resulting in a single frame for subsequent analyses.

We calculated $5 \sigma$ detection limit as follows. We defined a series of concentric annuli centering on the star. For the concentric annuli, we calculated the median and the standard deviation of flux for pixels within these annuli. We used the value of five times the standard deviation above the median as the $5 \sigma$ detection limit. The detection limits at different angular separations are reported in Table 1 . We developed an automatic program to detect stellar companions whose differential magnitudes are brighter than the $5 \sigma$ detection limit. The program recorded the differential magnitude, position, position angle, and detection significance of each detection. All detections were then visually checked to remove confusions such as speckles, background extended sources, and cosmic-ray hits. In total, 42 stellar companions were detected within $5^{\prime \prime}$ around 35 KOIs. Their properties are summarized in Table 2. Figure 1 shows nine KOIs with newly detected stellar companions within $2^{\prime \prime}$.

\subsection{Physical Association}

For stellar companions detected by imaging techniques, we need to check whether they are optical doubles/multiples, which will systematically increase the stellar multiplicity rate. To test physical association, Ngo et al. (2015) obtained multiple-epoch AO images and measured common proper motion. In our case, Kepler stars are generally farther away and common proper motion is more difficult to measure. Given only one epoch of observation, we can use color information of detected stellar companions and assess the probability of their physical association to primary stars (Lillo-Box et al. 2014; Wang et al. 2014a, 2015). The color information provides an estimate of the stellar properties, which can then be used to estimate distance for consistency check between the primary and the secondary stars. Any inconsistent distance would be an indication that the primary and the secondary stars are optical doubles. For stellar companions with only single-band observations, color information is not available. We can assess the probability with a galactic stellar population simulation. This method is described in detail in Wang et al. (2015), and the physical association probabilities of each detected stellar companions are given in Table 2.

\subsection{Combining AO Observations with Other Techniques}

Following the method described in Wang et al. (2015), we conduct simulations to estimate the search completeness for the $\mathrm{AO}$ observations. In these simulations, we use the AO contrast curve as a threshold for detection. In practice, however, not all stars above the $\mathrm{AO}$ contrast curve are detected by our pipeline, so we run another simulation to test the goodness of using the contrast curve as a threshold. The simulation is identical to other studies (Lillo-Box et al. 2014; Gilliland et al. 2015; Ngo et al. 2015) that artificially inject companion stars with the same PSF at random separations, differential magnitudes, and position angles. The results are shown in Figure 2 for two 

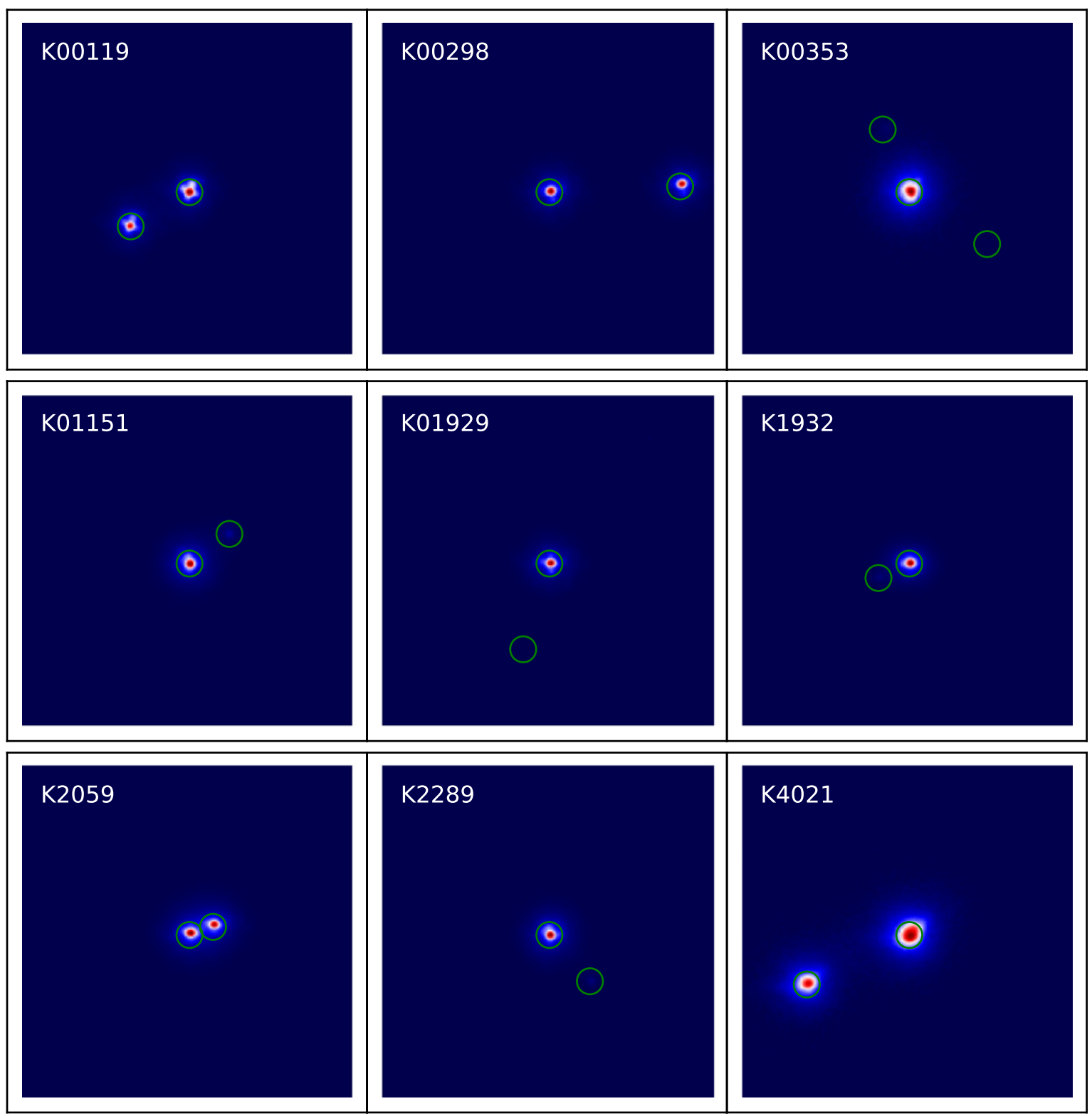

Figure 1. AO images for nine KOIs with newly detected stellar companions within $2^{\prime \prime}$. All images cover a $2^{\prime \prime}$ by $2^{\prime \prime}$ sky region centering at the primary star. North is up, and east is to the left. Linear colorscale is chosen such that the central star (red) is normalized to 1 and the background (blue) represents $1 / 100$ of the central star flux. Both central stars and detected stellar companions are marked by green circles. Photometric and astrometric information of detected stellar companions can be found in Table 2.

examples, one for a Palomar AO image and the other one for Keck. For the Palomar AO image, $94.7 \%$ of injected companion stars above the contrast curve are successfully recovered by our detection pipeline and $88.2 \%$ of injections below the contrast curve are missed. For the Keck image, $90.7 \%$ of injections are recovered above the contrast curve and $88.4 \%$ are missed below the contrast curve. The simulation shows that using the contrast curve as a detection threshold is a reasonable assumption. The resulting AO search completenesses are within a few percent for the case of using the $\mathrm{AO}$ contrast curve as a hard limit for detection and for the case using the artificial PSF injection result (Lillo-Box et al. 2014; Gilliland et al. 2015; Ngo et al. 2015). The comparable results are due to a relatively smooth distribution of masses and separations of stellar companions, which translates to a smooth distribution on the $\Delta \mathrm{Mag}$ - angular separation plane as shown in Figure 2. The hard-edge effect of using the AO contrast curve is averaged out and becomes comparable with a more realistic artificial PSF injection simulation.

Since AO imaging technique is not sensitive to stellar companions within or close to the diffraction limit of a telescope, we use other techniques to constrain the presence of stellar companions, i.e., the RV technique and the dynamical analysis (Wang et al. 2014b). There are 22 KOIs in our sample with at least three epochs of RV observation. Following the description of Wang et al. (2014a), we use the Keplerian Fitting Made Easy package (Giguere et al. 2012) to analyze the RV data. Among 22 KOIs with RV data, only KOI-5 exhibits an $\mathrm{RV}$ trend. The stellar companion that can potentially induce the trend is constrained to be beyond 7 AU (Wang et al. 2014a). More recent RV data suggest that in addition to two transiting planet candidates, two more distant components exist in the KOI-5 system (H. Isaacson 2015, private communication). One is a sub-stellar companion with a period of $\sim 2700$ days and the 

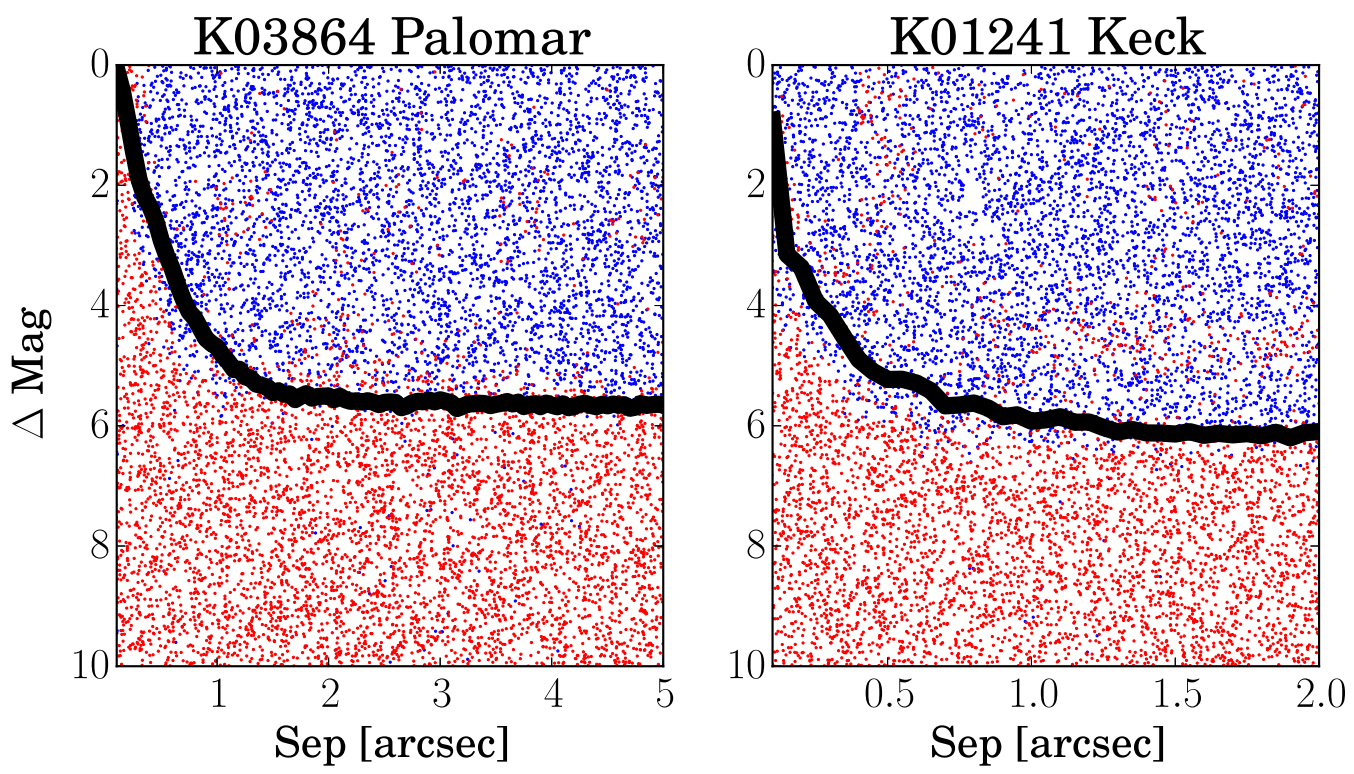

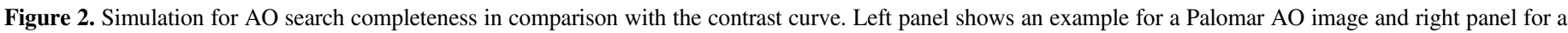

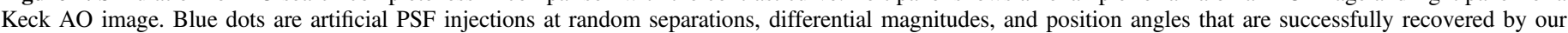


blue and red dots.

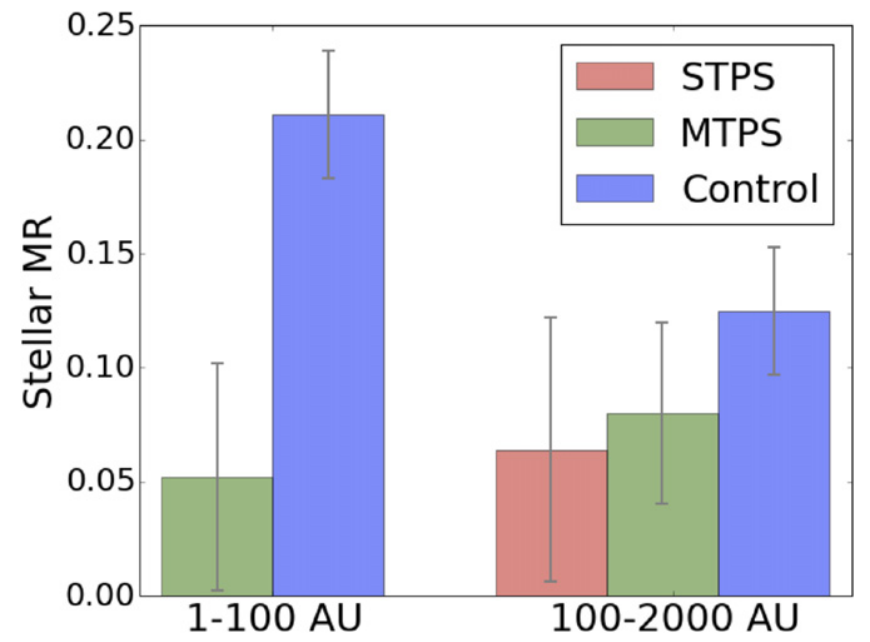

Figure 3. Stellar multiplicity rate for multiple transiting planet systems (MTPSs, green) and single transiting planet systems (STPSs, red), and the field stars in the solar neighborhood, i.e., the control sample in blue. The stellar multiplicity rates for different samples are given in Table 5.

other one is the AO-imaged stellar companion. Therefore, we consider the closest stellar companion to KOI-5 to have a projected separation of 40.12 AU (Table 2).

Besides RV and AO observations, we can use dynamical analysis to put additional constraints on potential stellar companions. This dynamical analysis makes use of the coplanarity of MTPSs discovered by the Kepler mission (Lissauer et al. 2011). A stellar companion with high mutual inclination to the planetary orbits would have perturbed the orbits and significantly reduced the coplanarity of planetary orbits, and hence the probability of multi-planet transits (see Section 2.6 in Wang et al. 2014b). Therefore, the fact that we have observed multiple transiting planet helps to exclude the possibility of a highly inclined stellar companion. The dynamical analysis is complementary to the RV technique because it is sensitive to stellar companions with large mutual inclinations to the planetary orbits. For systems with no stellar companions detected by the AO and/or RV method, an isolation probability can be calculated based on the search completeness of AO and RV observations and the constraints from the dynamical analysis (Wang et al. 2015). The isolation probability is a measure of how likely a star is isolated from other stellar companions within a certain distance. The isolation probabilities within $2000 \mathrm{AU}$ for KOIs with non-detections of stellar companions are given in Table 1.

\section{STELLAR MULTIPLICITY RATE FOR MTPSs}

Following the same method described in Wang et al. (2015), we calculate the stellar multiplicity rate for MTPSs as a function of $a$, i.e., the companion semimajor axis. We find that for $1 \mathrm{AU}<a<2000 \mathrm{AU}$, the stellar multiplicity rate for MTPSs is $13.3 \pm 5.7 \%$, which is significantly $(3.2 \sigma)$ lower than $33.6 \pm 2.8 \%$ for the control sample, i.e., the field stars in the solar neighborhood (Raghavan et al. 2010). We choose an upper limit of $2000 \mathrm{AU}$ for comparison because the separation roughly corresponds to the smallest FOV of co-added AO images, which have the best sensitivity for stellar companion search. We further divide the semimajor axis of a stellar companion into two ranges, $1 \mathrm{AU}<a<100 \mathrm{AU}$ and 100 AU $<a<2000$ AU. We choose 100 AU for two reasons. First, the separation is roughly the effective range of the perturbation of coplanarity by a companion star (see the discussion of Section 5.2). Second, $100 \mathrm{AU}$ is roughly the borderline of RV and AO sensitivity (Wang et al. 2014a, 2014b). Beyond $100 \mathrm{AU}$, the AO sensitivity is much higher than that for the RV technique. The stellar multiplicity rates for MTPSs are $5.2 \pm 5.0 \%$ and $8.0 \pm 4.0 \%$ for $1 \mathrm{AU}<a<100 \mathrm{AU}$ and $100 \mathrm{AU}<a<2000 \mathrm{AU}$, respectively. In comparison, the stellar multiplicity rates are $21.1 \pm$ $2.8 \%$ and $12.5 \pm 2.8 \%$ for the control sample in these two stellar separation ranges. The stellar multiplicity rate of MTPS for $1 \mathrm{AU}<a<100 \mathrm{AU}$ is lower $(2.8 \sigma)$ than that for the control sample. For $100 \mathrm{AU}<a<2000 \mathrm{AU}$, the stellar 
Table 3

Stellar Parameters for STPSs

\begin{tabular}{|c|c|c|c|c|c|c|c|}
\hline KOI & KIC & $\begin{array}{c}\alpha \\
\text { (h:m:s) }\end{array}$ & $\begin{array}{c}\delta \\
(\mathrm{d}: \mathrm{m}: \mathrm{s})\end{array}$ & $\begin{array}{c}K p \\
(\mathrm{mag})\end{array}$ & $\begin{array}{l}T_{\text {eff }} \\
(\mathrm{K})\end{array}$ & $\begin{array}{l}\log g \\
(\operatorname{cgs})\end{array}$ & $\begin{array}{c}{[\mathrm{Fe} / \mathrm{H}]} \\
(\mathrm{dex})\end{array}$ \\
\hline 00042 & 8866102 & $18: 52: 36.17$ & $45: 08: 23.4$ & 9.36 & 6325 & 4.26 & 0.01 \\
\hline 00069 & 3544595 & $19: 25: 40.39$ & $38: 40: 20.49$ & 9.93 & 5669 & 4.47 & -0.18 \\
\hline 00084 & 2571238 & $19: 21: 40.99$ & $37: 51: 06.48$ & 11.90 & 5543 & 4.57 & -0.14 \\
\hline 00087 & 10593626 & $19: 16: 52.2$ & 47:53:04.06 & 11.66 & 5642 & 4.44 & -0.27 \\
\hline 00092 & 7941200 & $18: 53: 29.96$ & $43: 47: 17.59$ & 11.67 & 5952 & 4.49 & -0.04 \\
\hline 00103 & 2444412 & $19: 26: 44$ & $37: 45: 05.73$ & 12.59 & 5653 & 4.55 & -0.06 \\
\hline 00118 & 3531558 & $19: 09: 27.07$ & $38: 38: 58.56$ & 12.38 & 5747 & 4.18 & 0.03 \\
\hline 00122 & 8349582 & $18: 57: 55.79$ & $44: 23: 52.95$ & 12.35 & 5699 & 4.17 & 0.30 \\
\hline 00180 & 9573539 & $18: 57: 34.63$ & $46: 14: 56.69$ & 13.02 & 5691 & 4.54 & -0.06 \\
\hline 00257 & 5514383 & $18: 58: 32.45$ & $40: 43: 11.39$ & 10.87 & 6184 & 4.36 & 0.12 \\
\hline 00261 & 5383248 & 19:48:16.71 & $40: 31: 30.47$ & 10.30 & 5763 & 4.53 & 0.04 \\
\hline 00265 & 12024120 & $19: 48: 04.52$ & $50: 24: 32.33$ & 11.99 & 6036 & 4.32 & 0.08 \\
\hline 00268 & 3425851 & 19:02:54.91 & $38: 30: 25.1$ & 10.56 & 6343 & 4.26 & -0.04 \\
\hline 00269 & 7670943 & 19:09:22.98 & $43: 22: 42.21$ & 10.93 & 6463 & 4.24 & 0.09 \\
\hline 00273 & 3102384 & 19:09:54.84 & $38: 13: 43.82$ & 11.46 & 5739 & 4.40 & 0.35 \\
\hline 00276 & 11133306 & $19: 18: 39.46$ & $48: 42: 22.36$ & 11.85 & 5982 & 4.32 & -0.02 \\
\hline 00280 & 4141376 & $19: 06: 45.47$ & $39: 12: 42.88$ & 11.07 & 6134 & 4.42 & -0.24 \\
\hline 00281 & 4143755 & $19: 10: 37.2$ & $39: 14: 39.44$ & 11.95 & 5622 & 4.09 & -0.40 \\
\hline 00292 & 11075737 & 19:09:18.39 & $48: 40: 24.35$ & 12.87 & 5802 & 4.42 & -0.20 \\
\hline 00299 & 2692377 & $19: 02: 38.8$ & $37: 57: 52.2$ & 12.90 & 5580 & 4.54 & 0.18 \\
\hline 00303 & 5966322 & $19: 34: 42.08$ & $41: 17: 43.3$ & 12.19 & 5598 & 4.32 & -0.12 \\
\hline 00306 & 6071903 & $19: 57: 16.69$ & $41: 23: 04.7$ & 12.63 & 5377 & 4.58 & 0.10 \\
\hline 00344 & 11015108 & $18: 53: 21.67$ & $48: 32: 56.55$ & 13.40 & 5957 & 4.35 & -0.04 \\
\hline 00364 & 7296438 & $19: 43: 29.36$ & $42: 52: 52.14$ & 10.09 & 5749 & 4.17 & -0.20 \\
\hline 00374 & 8686097 & $19: 22: 30.06$ & $44: 52: 26.25$ & 12.21 & 5839 & 4.20 & -0.22 \\
\hline 00974 & 9414417 & $19: 43: 12.64$ & 45:59:17.08 & 9.58 & 6253 & 4.00 & -0.13 \\
\hline 00975 & 3632418 & 19:09:26.84 & $38: 42: 50.46$ & 8.22 & 6131 & 4.03 & -0.15 \\
\hline 01162 & 10528068 & $19: 15: 28.37$ & $47: 45: 33.95$ & 12.78 & 6126 & 4.28 & -0.28 \\
\hline 01311 & 10713616 & $18: 54: 07.91$ & $48: 05: 39.34$ & 13.50 & 6190 & 4.18 & -0.10 \\
\hline 01442 & 11600889 & 19:04:08.72 & $49: 36: 52.24$ & 12.52 & 5626 & 4.40 & 0.34 \\
\hline 01537 & 9872292 & $18: 45: 50.82$ & $46: 47: 23.62$ & 11.74 & 6260 & 4.05 & 0.10 \\
\hline 01612 & 10963065 & 18:59:08.69 & $48: 25: 23.62$ & 8.77 & 6104 & 4.29 & -0.20 \\
\hline 01615 & 4278221 & $19: 41: 17.4$ & $39: 22: 35.37$ & 11.52 & 5977 & 4.47 & 0.21 \\
\hline 01618 & 7215603 & $19: 44: 11.37$ & $42: 44: 34.84$ & 11.60 & 6173 & 4.19 & 0.17 \\
\hline 01619 & 4276716 & $19: 39: 57.66$ & $39: 20: 46.96$ & 11.76 & 4827 & 4.60 & -0.34 \\
\hline 01808 & 7761918 & $19: 38: 58.4$ & $43: 27: 40.35$ & 12.49 & 6277 & 4.35 & -0.06 \\
\hline 01883 & 11758544 & $19: 16: 56.01$ & $49: 56: 20.15$ & 11.89 & 6287 & 4.34 & 0.02 \\
\hline 01890 & 7449136 & $19: 32: 19.08$ & $43: 04: 25.36$ & 11.70 & 6099 & 4.13 & 0.04 \\
\hline 01925 & 9955598 & $19: 34: 43.01$ & 46:51:09.94 & 9.44 & 5460 & 4.50 & 0.08 \\
\hline 01962 & 5513648 & $18: 56: 56.15$ & $40: 47: 40.34$ & 10.77 & 5904 & 4.13 & -0.07 \\
\hline 01964 & 7887791 & $19: 22: 48.89$ & $43: 36: 25.95$ & 10.69 & 5547 & 4.39 & -0.06 \\
\hline 02032 & 2985767 & $19: 22: 06.42$ & $38: 08: 34.72$ & 12.26 & 5568 & 4.50 & -0.04 \\
\hline 02087 & 6922710 & $18: 46: 14.75$ & $42: 27: 01.8$ & 11.86 & 5930 & 4.40 & 0.07 \\
\hline 02110 & 11460462 & $19: 37: 52.45$ & $49: 19: 51.67$ & 12.19 & 6452 & 4.37 & 0.21 \\
\hline 02215 & 7050060 & $19: 45: 01.22$ & $42: 31: 48.79$ & 13.00 & 5974 & 4.22 & -0.24 \\
\hline 02260 & 11811193 & $19: 20: 56.6$ & 50:01:48.32 & 12.17 & 6444 & 4.39 & 0.02 \\
\hline 02295 & 4049901 & $19: 18: 10.83$ & 39:09:51.94 & 11.67 & 5451 & 4.45 & -0.22 \\
\hline 02324 & 7746958 & $19: 18: 42.69$ & $43: 27: 29.28$ & 11.67 & 5780 & 4.44 & 0.00 \\
\hline 02462 & 5042210 & $19: 55: 58.01$ & $40: 08: 32.72$ & 11.82 & 6006 & 4.27 & 0.04 \\
\hline 02593 & 8212002 & $18: 47: 20.48$ & 44:09:21.3 & 11.71 & 6141 & 4.07 & 0.28 \\
\hline 02632 & 11337566 & $18: 57: 41.45$ & 49:06:22.39 & 11.39 & 6461 & 4.17 & 0.18 \\
\hline 02706 & 9697131 & 19:00:18.64 & $46: 25: 10.56$ & 10.27 & 6491 & 4.02 & -0.20 \\
\hline 02712 & 11098013 & $19: 50: 59.35$ & $48: 41: 39.51$ & 11.12 & 6450 & 4.26 & 0.32 \\
\hline 02720 & 8176564 & $19: 41: 45.52$ & 44:02:20.98 & 10.34 & 6109 & 4.14 & -0.20 \\
\hline 02754 & 10905911 & $18: 54: 59$ & $48: 22: 24.36$ & 12.30 & 5738 & 4.11 & -0.08 \\
\hline 02790 & 5652893 & $19: 58: 38.31$ & $40: 50: 37.86$ & 13.38 & 5153 & 4.55 & -0.18 \\
\hline 02792 & 11127479 & $19: 05: 21.2$ & $48: 44: 38.76$ & 11.13 & 5998 & 4.22 & -0.20 \\
\hline 02904 & 3969687 & $19: 41: 30.57$ & 39:02:52.91 & 12.68 & 6046 & 4.48 & 0.36 \\
\hline 02948 & 6356692 & $19: 17: 34.74$ & $41: 46: 56.46$ & 11.93 & 5675 & 4.03 & 0.00 \\
\hline 02968 & 8873090 & 19:06:19.23 & 45:09:49.76 & 11.91 & 6387 & 4.28 & -0.14 \\
\hline 03008 & 9070666 & 18:50:47.99 & $45: 25: 32.77$ & 12.00 & 6295 & 4.28 & -0.14 \\
\hline 03122 & 12416661 & $19: 42: 09.21$ & $51: 12: 10.66$ & 12.09 & 6350 & 4.15 & 0.24 \\
\hline 03165 & 9579208 & $19: 10: 33.02$ & $46: 12: 15.88$ & 10.34 & 6422 & 4.02 & -0.20 \\
\hline
\end{tabular}


Table 3

(Continued)

\begin{tabular}{|c|c|c|c|c|c|c|c|}
\hline KOI & KIC & $\begin{array}{c}\alpha \\
\text { (h:m:s) }\end{array}$ & $\begin{array}{c}\delta \\
(\mathrm{d}: \mathrm{m}: \mathrm{s})\end{array}$ & $\begin{array}{c}K p \\
(\mathrm{mag})\end{array}$ & $\begin{array}{l}T_{\text {eff }} \\
(\mathrm{K})\end{array}$ & $\begin{array}{l}\log g \\
(\operatorname{cgs})\end{array}$ & $\begin{array}{c}{[\mathrm{Fe} / \mathrm{H}]} \\
(\mathrm{dex})\end{array}$ \\
\hline 03168 & 4450844 & 19:09:15.56 & $39: 32: 17.45$ & 10.46 & 5968 & 4.09 & -0.20 \\
\hline 03179 & 6153407 & $19: 57: 12.67$ & $41: 26: 27.66$ & 10.88 & 6237 & 4.03 & 0.00 \\
\hline 03190 & 5985713 & $19: 53: 04.36$ & $41: 15: 05.99$ & 11.46 & 6280 & 4.35 & -0.22 \\
\hline 03225 & 3109550 & $19: 18: 41.22$ & $38: 17: 52.34$ & 12.21 & 5511 & 4.13 & 0.06 \\
\hline 03234 & 10057494 & $18: 53: 44.58$ & 47:04:00.7 & 12.28 & 6379 & 4.36 & 0.00 \\
\hline 03245 & 8073705 & $18: 40: 59.87$ & $43: 54: 54.21$ & 12.40 & 6086 & 4.37 & -0.16 \\
\hline 03248 & 10917433 & $19: 21: 51.62$ & 48:19:56.1 & 12.42 & 5680 & 4.32 & 0.00 \\
\hline 03880 & 4147444 & $19: 15: 28.17$ & $39: 15: 53.86$ & 10.76 & 6438 & 4.33 & -0.26 \\
\hline 03946 & 8636434 & $19: 43: 54.13$ & $44: 42: 48.42$ & 13.21 & 6363 & 4.44 & -0.26 \\
\hline 04160 & 7610663 & 19:31:08.31 & $43: 12: 57.53$ & 13.42 & 5755 & 4.40 & -0.14 \\
\hline 04329 & 12456063 & $19: 16: 02.83$ & $51: 22: 33.67$ & 12.02 & 6338 & 4.45 & 0.14 \\
\hline 04407 & 8396660 & 20:04:37.57 & $44: 22: 46.32$ & 11.18 & 6331 & 4.09 & 0.20 \\
\hline 04409 & 5308537 & 19:58:08.35 & $40: 28: 40$ & 12.52 & 5826 & 4.28 & 0.14 \\
\hline 04582 & 7905106 & $19: 45: 20.85$ & $43: 36: 00.32$ & 11.76 & 5984 & 4.05 & -0.20 \\
\hline 04878 & 11804437 & 19:04:54.75 & $50: 00: 48.89$ & 12.29 & 6031 & 4.37 & -0.22 \\
\hline 05068 & 4484179 & $19: 45: 41.45$ & $39: 34: 45.81$ & 13.09 & 6440 & 4.36 & -0.76 \\
\hline 05087 & 4770798 & $19: 50: 02.2$ & $39: 53: 16.87$ & 12.52 & 5696 & 4.22 & 0.04 \\
\hline 05236 & 6067545 & $19: 53: 35.52$ & 41:18:53.61 & 13.09 & 6241 & 4.45 & -0.14 \\
\hline 05254 & 6266866 & 18:58:21.99 & $41: 38: 21.38$ & 10.93 & 5807 & 4.11 & 0.06 \\
\hline 05556 & 8656535 & 20:06:01.57 & $44: 42: 42.63$ & 13.41 & 5594 & 4.39 & 0.00 \\
\hline 05665 & 9394953 & $19: 09: 25.15$ & $45: 56: 55.18$ & 11.48 & 6018 & 4.04 & -0.20 \\
\hline 05806 & 10552263 & $19: 51: 28.81$ & $47: 46: 15.93$ & 12.36 & 5914 & 4.45 & -0.12 \\
\hline 05833 & 10850327 & $19: 06: 21.89$ & $48: 13: 12.96$ & 13.01 & 6277 & 4.43 & -0.46 \\
\hline 05938 & 11860294 & $19: 18: 36.83$ & $50: 07: 40.84$ & 12.81 & 6273 & 4.34 & -0.08 \\
\hline 05949 & 12009917 & $19: 18: 44.52$ & $50: 24: 33.22$ & 13.29 & 6201 & 4.35 & -0.20 \\
\hline 06108 & 4139254 & 19:03:27.05 & $39: 12: 19.01$ & 12.12 & 5551 & 4.39 & -0.22 \\
\hline 06202 & 9389245 & $18: 56: 33.87$ & $45: 56: 40.71$ & 11.54 & 6021 & 4.13 & -0.54 \\
\hline 06246 & 11856178 & $19: 08: 39.61$ & $50: 06: 47.64$ & 11.77 & 6122 & 4.49 & -0.18 \\
\hline
\end{tabular}

multiplicity rates are comparable between MTPS and the control sample. Figure 3 illustrates the comparison of the stellar multiplicity rates in these two separation ranges.

\section{DISCUSSION AND SUMMARY}

\subsection{Interpretation of the Stellar Multiplicity of MTPSs}

The stellar multiplicity rate for MTPSs $(5.2 \pm 5.0 \%)$ is $2.8 \sigma$ lower than that for stars in the solar neighborhood $(21.1 \pm$ $2.8 \%$ ) for $1 \mathrm{AU}<a<100 \mathrm{AU}$. The difference may result from two possible origins that are not mutually exclusive. First, MTPSs occur less frequently in multiple stellar systems. Suppressive planet formation in multiple stellar systems has been noted in previous observational works on both RV and transiting planet samples (e.g., Eggenberger et al. 2011; Roell et al. 2012; Wang et al. 2014b) and recently a theoretical work (Touma \& Sridhar 2015). However, other works suggest that the influence of a stellar companion may not be significant (Horch et al. 2014; Gilliland et al. 2015) or may be facilitative depending on the stellar separation and planetary mass (Ngo et al. 2015; Wang et al. 2015).

If suppressive planet formation does not play a role, there may be another origin for the low stellar multiplicity rate: MTPSs are less likely to be observed in multiple stellar systems (Wang et al. 2014b). Coplanarity of MTPSs can be affected by an additional stellar component. Thus, the likelihood of observing multiple transiting planets is reduced.

If suppressive planet formation plays a major role, then our measurements of stellar multiplicity rates indicate that within $100 \mathrm{AU}, \mathrm{MTPSs}$ occur less frequently due to the influence of stellar companions. For $100 \mathrm{AU}<a<2000 \mathrm{AU}$, since the stellar multiplicity rates are comparable $(0.9 \sigma$ difference) between MTPSs $(8.0 \pm 4.0 \%)$ and the control sample $(12.5$ $\pm 2.8 \%$ ), we conclude that the influence of stellar companions, if any, is too small to be observed.

\subsection{Comparison to STPSs}

If coplanarity is responsible for the observed low stellar multiplicity rate for MTPSs, then we should expect a difference of stellar multiplicity rate between MTPSs and STPSs. Note that the influence of stellar companions on coplanarity depends on stellar separations. If stellar separations are beyond $\sim 100 \mathrm{AU}$, their influence on coplanarity is negligible (Wang et al. 2014a, 2014b). Therefore, any difference of the stellar multiplicity rate beyond $100 \mathrm{AU}$ is more likely to be due to the origin of planet formation rather than the companions' influence on coplanarity.

In Section 5.1, we show that beyond $100 \mathrm{AU}$, the stellar multiplicity rates are comparable between MTPSs and the control sample. Here, we compare MTPSs to STPSs. Since these two populations likely have different dynamical histories (Morton \& Winn 2014; Xie et al. 2014), the comparison allows us to study whether the difference is related to stellar multiplicity.

From CFOP, we select 89 Kepler STPSs. The selection criteria are the same as described in Section 2 with two exceptions: (1) the number of transiting planets is equal to one; (2) they must have AO images. The stellar properties of these STPSs are given in Table 3. The sample of these STPSs is a subsample of Kepler stars with high-resolution imaging observations from CFOP (D. Ciardi 2015, in preparation). 
Table 4

Visual Companion Detections with AO Data for Kepler STPS

\begin{tabular}{|c|c|c|c|c|c|c|c|c|c|c|c|}
\hline \multirow{2}{*}{ KOI } & \multirow{2}{*}{ Star\# } & \multirow{2}{*}{ Telescope } & \multirow{2}{*}{ Filter } & \multirow{2}{*}{$\begin{array}{c}\Delta \mathrm{Mag}^{\mathrm{a}} \\
(\mathrm{mag})\end{array}$} & \multicolumn{2}{|c|}{ Separation $^{\mathrm{b}}$} & \multicolumn{2}{|c|}{ Distance $^{c}$} & \multirow{2}{*}{$\begin{array}{c}\text { PA } \\
(\mathrm{deg})\end{array}$} & \multirow{2}{*}{$\begin{array}{l}\text { Association }^{\mathrm{d}} \\
\text { Probability }\end{array}$} & \multirow[t]{2}{*}{ Ref. $^{e}$} \\
\hline & & & & & $(\operatorname{arcsec})$ & $(\mathrm{AU})$ & $\begin{array}{l}\text { Primary } \\
(\mathrm{pc})\end{array}$ & $\begin{array}{l}\text { Secondary } \\
\text { (pc) }\end{array}$ & & & \\
\hline K00118 & 1 & Palomar & $\mathrm{J}$ & 3.94 & 1.24 & 583.76 & $470.3_{-24.4}^{18.8}$ & $1152.1_{-605.8}^{878.0}$ & 214.3 & $>0.90$ & CFOP \\
\hline K00118 & 1 & Palomar & $\mathrm{K}$ & 3.65 & 1.23 & 578.94 & $470.3_{-24.4}^{18.8}$ & $1152.1_{-605.8}^{878.0}$ & 214.6 & $>0.90$ & CFOP \\
\hline K00268 & 1 & MMT & $\mathrm{J}$ & 3.03 & 1.57 & 372.64 & $238.1_{-7.1}^{32.6}$ & $305.4_{-274.7}^{116.2}$ & 179.7 & $>0.90$ & CFOP \\
\hline K00268 & 1 & MMT & $\mathrm{K}$ & 2.52 & 1.65 & 392.07 & $238.1_{-7.1}^{32.6}$ & $305.4_{-274.7}^{116.2}$ & 174.8 & $>0.90$ & $\mathrm{CFOP}$ \\
\hline K00268 & 1 & Palomar & K & 2.47 & 1.75 & 415.62 & $238.1_{-7.1}^{32.6}$ & $305.4_{-274.7}^{116.2}$ & 267.3 & $>0.90$ & CFOP \\
\hline K00268 & 2 & MMT & $\mathrm{J}$ & 4.37 & 2.34 & 556.29 & $238.1_{-7.1}^{32.6}$ & $305.4_{-274.7}^{116.2}$ & 128.1 & $>0.90$ & CFOP (Dupree) \\
\hline K00268 & 2 & MMT & K & 3.87 & 2.33 & 554.58 & $238.1_{-7.1}^{32.6}$ & $305.4_{-274.7}^{116.2}$ & 132.0 & $>0.90$ & CFOP (Dupree) \\
\hline K00268 & 2 & Palomar & $\mathrm{K}$ & 3.72 & 2.49 & 593.65 & $238.1_{-7.1}^{32.6}$ & $305.4_{-274.7}^{116.2}$ & 309.9 & $>0.90$ & CFOP \\
\hline K00273 & 1 & MMT & $\mathrm{J}$ & 4.75 & 0.51 & 122.11 & $239.0_{-12.0}^{11.5}$ & $25619.3_{-14890.6}^{15172.9}$ & 152.4 & $>0.90$ & CFOP (Dupree) \\
\hline K00273 & 1 & MMT & $\mathrm{K}$ & 5.31 & 0.55 & 131.77 & $239.0_{-12.0}^{11.5}$ & $25619.3_{-14890.6}^{15172.9}$ & 152.4 & $>0.90$ & CFOP (Dupree) \\
\hline K00306 & 1 & Palomar & $\mathrm{J}$ & 2.27 & 2.08 & 473.60 & $228.2_{-8.6}^{9.2}$ & $400.2_{-348.9}^{189.0}$ & 245.4 & $>0.90$ & CFOP \\
\hline K00306 & 1 & Palomar & K & 1.95 & 2.08 & 475.48 & $228.2_{-8.6}^{9.2}$ & $400.2_{-348.9}^{189.0}$ & 245.3 & $>0.90$ & $\mathrm{CFOP}$ \\
\hline K00344 & 1 & Palomar & $\mathrm{K}$ & 3.53 & 4.13 & 2465.18 & $597.5_{-126.2}^{290.0}$ & $\ldots$ & 178.8 & 0.76 & this work \\
\hline K00344 & 2 & Palomar & $\mathrm{K}$ & 5.30 & 3.57 & 2132.69 & $597.5_{-126.2}^{290.0}$ & $\ldots$ & 210.5 & 0.39 & this work \\
\hline K00374 & 1 & Palomar & $\mathrm{J}$ & 6.03 & 1.76 & 643.62 & $366.6_{-28.1}^{124.0}$ & $20614.0_{-12594.6}^{4747.0}$ & 88.3 & 0.69 & CFOP \\
\hline K00374 & 1 & Palomar & K & 6.32 & 1.85 & 676.52 & $366.6_{-28.1}^{124.0}$ & $20614.0_{-12594.6}^{4747.0}$ & 87.4 & 0.67 & CFOP \\
\hline K01311 & 1 & Palomar & K & 4.20 & 0.44 & 284.23 & $648.2_{-111.1}^{483.8}$ & $\cdots$ & 175.9 & $>0.90$ & this work \\
\hline K01537 & 1 & MMT & K & 0.13 & 0.09 & 45.56 & $522.5_{-56.1}^{28.3}$ & $\ldots$ & 64.5 & $>0.90$ & CFOP (Dupree) \\
\hline K01615 & 1 & Palomar & $\mathrm{K}$ & 6.60 & 2.98 & 610.53 & $205.1_{-11.7}^{13.5}$ & $\ldots$ & 357.8 & 0.18 & CFOP \\
\hline K01619 & 1 & Keck & $\mathrm{K}$ & 2.00 & 2.09 & 265.00 & $126.8_{-10.9}^{4.3}$ & $\ldots$ & 226.7 & $>0.90$ & CFOP \\
\hline K01808 & 1 & Palomar & K & 3.30 & 4.69 & 1991.97 & $424.4_{-70.8}^{177.3}$ & $\cdots$ & 162.9 & 0.66 & this work \\
\hline K01890 & 1 & Keck & K & 2.02 & 0.41 & 181.54 & $443.0_{-45.5}^{13.5}$ & $\ldots$ & 145.4 & $>0.90$ & CFOP \\
\hline K01964 & 1 & Palomar & $\mathrm{J}$ & 2.09 & 0.40 & 51.28 & $129.2_{-13.0}^{14.4}$ & $186.2_{-152.8}^{127.1}$ & 0.4 & $>0.90$ & $\mathrm{CFOP}$ \\
\hline K01964 & 1 & Palomar & $\mathrm{K}$ & 1.83 & 0.40 & 51.28 & $129.2_{-13.0}^{14.4}$ & $186.2_{-152.8}^{127.1}$ & 0.9 & $>0.90$ & CFOP \\
\hline K02032 & 1 & Palomar & K & 0.40 & 1.10 & 311.71 & $283.8_{-27.0}^{19.2}$ & $\ldots$ & 311.4 & $>0.90$ & CFOP \\
\hline K02324 & 1 & Palomar & K & 0.48 & 4.73 & 7271.72 & $1537.1_{-258.9}^{1574.8}$ & $\cdots$ & 353.4 & 0.73 & CFOP \\
\hline K02706 & 1 & Palomar & K & 5.37 & 1.66 & 455.08 & $273.7_{-21.3}^{27.1}$ & $\ldots$ & 165.8 & $>0.90$ & CFOP \\
\hline K02754 & 1 & Palomar & K & 1.55 & 0.79 & 231.80 & $294.9_{-35.4}^{296.7}$ & $\ldots$ & 260.4 & $>0.90$ & CFOP \\
\hline K02790 & 1 & Keck & $\mathrm{K}$ & 0.48 & 0.26 & 88.75 & $341.5_{-28.8}^{16.7}$ & $\ldots$ & 134.6 & $>0.90$ & CFOP \\
\hline K02904 & 1 & Palomar & K & 2.16 & 0.69 & 264.31 & $383.2_{-27.2}^{33.8}$ & $\ldots$ & 226.4 & $>0.90$ & CFOP \\
\hline K03168 & 1 & Palomar & $\mathrm{J}$ & 3.78 & 0.80 & 192.09 & $239.4_{-22.9}^{8.0}$ & $379.0_{-334.5}^{132.3}$ & 332.6 & $>0.90$ & $\mathrm{CFOP}$ \\
\hline K03168 & 1 & Keck & K & 3.37 & 0.81 & 193.33 & $239.4_{-22.9}^{8.0}$ & $379.0_{-334.5}^{132.3}$ & 332.3 & $>0.90$ & CFOP \\
\hline K03168 & 1 & Palomar & $\mathrm{K}$ & 3.33 & 0.81 & 192.81 & $239.4_{-22.9}^{8.0}$ & $379.0_{-334.5}^{132.3}$ & 332.2 & $>0.90$ & CFOP \\
\hline K03190 & 1 & Palomar & K & 3.96 & 2.38 & 954.33 & $401.7_{-57.8}^{56.7}$ & $\ldots$ & 188.4 & 0.90 & CFOP \\
\hline K03245 & 1 & Palomar & $\mathrm{K}$ & 1.84 & 1.54 & 590.39 & $384.0_{-27.1}^{54.1}$ & $\ldots$ & 185.1 & $>0.90$ & CFOP \\
\hline K03248 & 1 & Palomar & K & 4.76 & 3.98 & 1332.34 & $334.7_{-37.3}^{53.4}$ & $\ldots$ & 242.5 & 0.48 & CFOP \\
\hline K04329 & 1 & Keck & K & 2.89 & 1.84 & 625.41 & $340.0_{-33.3}^{26.0}$ & $\ldots$ & 118.6 & $>0.90$ & CFOP \\
\hline
\end{tabular}


Table 4

(Continued)

\begin{tabular}{|c|c|c|c|c|c|c|c|c|c|c|c|}
\hline \multirow{2}{*}{ KOI } & \multirow{2}{*}{ Star\# } & \multirow{2}{*}{ Telescope } & \multirow{2}{*}{ Filter } & \multirow{2}{*}{$\begin{array}{c}\Delta \mathrm{Mag}^{\mathrm{a}} \\
(\mathrm{mag})\end{array}$} & \multicolumn{2}{|c|}{ Separation $^{\mathrm{b}}$} & \multicolumn{2}{|c|}{ Distance $^{c}$} & \multirow{2}{*}{$\begin{array}{c}\text { PA } \\
(\mathrm{deg})\end{array}$} & \multirow{2}{*}{$\begin{array}{l}\text { Association }^{\mathrm{d}} \\
\text { Probability }\end{array}$} & \multirow{2}{*}{ Ref. $^{\text {e }}$} \\
\hline & & & & & $(\operatorname{arcsec})$ & (AU) & $\begin{array}{l}\text { Primary } \\
\text { (pc) }\end{array}$ & $\begin{array}{l}\text { Secondary } \\
\text { (pc) }\end{array}$ & & & \\
\hline K04407 & 1 & Palomar & $\mathrm{K}$ & 1.99 & 2.46 & 616.94 & $251.0_{-37.6}^{193.0}$ & $\ldots$ & 299.9 & $>0.90$ & CFOP \\
\hline K04407 & 2 & Palomar & $\mathrm{K}$ & 4.91 & 2.65 & 665.76 & $251.0_{-37.6}^{193.0}$ & $\ldots$ & 311.2 & 0.84 & CFOP \\
\hline K05236 & 1 & Palomar & K & 6.01 & 1.93 & 966.01 & $500.5_{-41.8}^{41.3}$ & $\ldots$ & 281.9 & 0.44 & CFOP \\
\hline K05556 & 1 & Palomar & $\mathrm{K}$ & 2.70 & 3.33 & 1300.46 & $391.1_{-48.8}^{54.3}$ & $\ldots$ & 162.7 & $>0.90$ & CFOP \\
\hline K05556 & 2 & Palomar & K & 3.97 & 3.15 & 1233.77 & $391.1_{-48.8}^{54.3}$ & $\ldots$ & 248.6 & 0.83 & CFOP \\
\hline K05665 & 1 & Palomar & K & 2.27 & 2.08 & 847.21 & $407.2_{-54.6}^{12.6}$ & $\ldots$ & 94.1 & $>0.90$ & CFOP \\
\hline K05949 & 1 & Palomar & K & 3.06 & 0.69 & 415.34 & $600.9_{-86.5}^{74.7}$ & $\ldots$ & 255.3 & $>0.90$ & CFOP \\
\hline
\end{tabular}

Notes.

${ }^{\text {a }}$ Typical $\Delta$ Mag uncertainty is $0.1 \mathrm{mag}$. The uncertainty is estimated from the companion injection simulation described in Section 3.3 .

b Typical angular separation uncertainty is 0 ." 05 . The uncertainty is estimated from the companion injection simulation described in Section 3.3 .

${ }^{c}$ Distance is estimated based on stellar properties of primary stars (Huber et al. 2014) and color information of secondary stars (see Section 4.1 in Wang et al. 2015 for more details).

${ }^{\mathrm{d}}$ Association probability has $10 \%$ uncertainty due to statistical error in simulation.

e AO images from CFOP are provided by David Ciardi unless otherwise noted.

Table 5

Stellar Multiplicity Rate within a Certain Stellar Separation for MTPSs, STPSs, and Field Stars in the Solar Neighborhood (i.e., the Control Sample)

\begin{tabular}{lcccccc}
\hline \hline$a$ & \multicolumn{2}{c}{ MTPS } & \multicolumn{2}{c}{ STPS } & \multicolumn{2}{c}{ Control Sample } \\
$(\mathrm{AU})$ & $\mathrm{MR}$ & $\delta \mathrm{MR}$ & $\mathrm{MR}$ & $\delta \mathrm{MR}$ & $\mathrm{MR}$ & $\delta \mathrm{MR}$ \\
\hline $1<a<100$ & 0.052 & 0.050 & $\ldots$ & $\ldots$ & 0.211 & 0.028 \\
$100<a<2000$ & 0.080 & 0.040 & 0.064 & 0.058 & 0.125 & 0.028 \\
\hline
\end{tabular}

Out of these 89 Kepler stars, only 6 have RV observations. Since the RV technique is sensitive to close-in stellar companions, obtaining the statistics for stellar companions within $100 \mathrm{AU}$ is difficult. Therefore, we focus on $100 \mathrm{AU}<a<2000 \mathrm{AU}$. The AO detections are listed in Table 4. Following the same method in Wang et al. (2015), we find that the stellar multiplicity rate is $6.4 \pm 5.8 \%$ for STPSs for $100 \mathrm{AU}<a<2000 \mathrm{AU}$. The value is consistent with that for MTPSs, i.e., $8.0 \pm 4.0 \%$. Therefore, we find no evidence that stellar companions between 100 and 2000 AU are responsible for the difference of orbital configuration between MTPSs and STPSs. However, the difference may be caused by stellar companions within $100 \mathrm{AU}$, for which we do not have adequate observational constraints.

\subsection{Comparison to Previous Result}

The same sample of 138 MTPSs were studied in Wang et al. (2014b). They found evidence of suppressive planet formation in tight binary stellar systems with $a<20 \mathrm{AU}$. This finding is consistent with the finding in this paper that the stellar multiplicity rate for MTPSs is lower than the control sample within $100 \mathrm{AU}$ at the $2.8 \sigma$ level. However, we cannot rule out another possibility that may cause the low stellar multiplicity, i.e., the influence of stellar companions on coplanarity of planetary orbits.

Combining newly obtained AO imaging data with archival $\mathrm{RV}$ data, we improve the statistics of stellar companions of planet host stars at large semimajor axes. For example, in Wang et al. (2014b), stellar multiplicity rate can only be constrained within $\sim 100$ AU because of a lack of AO imaging data. In this work, we extend the constraints to 2000 AU. Even within $100 \mathrm{AU}$, the stellar companion statistics is improved by the AO imaging data. This is because the AO imaging technique complements the RV technique at semimajor axes at which the dynamical signals are difficult to detect. The combination of $\mathrm{AO}$ and RV data enables the detection of a deficit of stellar companions to MTPSs within 100 AU.

Wang et al. (2014a) combined RV and AO data for 56 Kepler planet host stars. The stellar multiplicity rate for $a<2000 \mathrm{AU}$ was $43.2 \pm 5.7 \%$, which is a factor of three higher than what we reported in this paper, i.e., $13.3 \pm 5.7 \%$. The discrepancy is due to two reasons. First, we exclude optical doubles, whereas Wang et al. (2014a) included both optical doubles and physically associated companions. Physical separation of $2000 \mathrm{AU}$ roughly corresponds to $3^{\prime \prime}-6^{\prime \prime}$ angular separation (for the typical distances to these Kepler stars), at which the physical association probability is $\sim 50 \%$. Therefore, roughly half of the visual companions are expected to be optical doubles around 2000 AU. Second, we considered statistics of stellar companions to planet host stars when calculating the incompleteness of the companion search (Wang et al. 2015). In comparison, Wang et al. (2014a) considered statistics of stellar companions for stars in the solar neighborhood. The companion search incompleteness was overestimated in Wang et al. (2014a) because the stellar multiplicity rate for planet host stars is generally lower than that for stars in the solar neighborhood, especially for small semimajor axes. Therefore, the correction factor due to search incompleteness is smaller, resulting in a lower stellar multiplicity rate.

\subsection{Summary and Conclusion}

We study the influence of stellar companions on MTPSs using a sample of 138 Kepler MTPSs. We search for stellar 
companions to these planet host stars with $\mathrm{AO}$ images and archival RV data. In total, we detected 42 stellar companions within $5^{\prime \prime}$ around 35 multi-planet host stars. The properties of detected stellar companions are summarized in Table 2 . We also provide detection limits for all stars in our sample in Table 1.

We compare the stellar multiplicity rate between MTPSs and a control sample, i.e., stars in the solar neighborhood. For semimajor axes $1 \mathrm{AU}<a<2000 \mathrm{AU}$, the stellar multiplicity rate is $13.3 \pm 5.7 \%$ for MTPSs, which is $3.2 \sigma$ lower than 33.6 $\pm 2.8 \%$ for the control sample, i.e., the field stars in the solar neighborhood (Raghavan et al. 2010). The deficit of stellar companions to MTPSs can be a result of two origins, a suppressive planet formation and the disruption of coplanarity due to stellar companions. Since the latter may only be effective within $100 \mathrm{AU}$, we divide the semimajor axes into two ranges, $1 \mathrm{AU}<a<100 \mathrm{AU}$ and $100 \mathrm{AU}<a<2000 \mathrm{AU}$. The stellar multiplicity rate of MTPSs for $1 \mathrm{AU}<a<100 \mathrm{AU}$ is lower $(2.8 \sigma)$ than that for the control sample. The stellar multiplicity rates are comparable between MTPSs and the control sample for $100 \mathrm{AU}<a<2000 \mathrm{AU}$.

We also compare the stellar multiplicity rates for MTPSs and STPSs. No quantitative difference is found between MTPSs and STPSs for $100 \mathrm{AU}<a<2000 \mathrm{AU}$. For $1 \mathrm{AU}<a<100 \mathrm{AU}$, our data are insufficient for comparative study between MTPSs and STPSs because of a lack of RV data for STPSs. Based on these results, we cannot distinguish the two origins that could be responsible for the low stellar multiplicity rate for MTPSs for 1 AU $<a<100$ AU. Future $\mathrm{AO}$ and $\mathrm{RV}$ follow-up observations for a larger sample are needed for such a comparative study between MTPSs and STPSs.

The authors thank the anonymous referee for constructive comments and suggestions that greatly improved the paper. We would like to thank the telescope operators and supporting astronomers at the Palomar Observatory and the Keck Observatory. Some of the data presented herein were obtained at the W.M. Keck Observatory, which is operated as a scientific partnership among the California Institute of Technology, the University of California, and the National Aeronautics and Space Administration. The Observatory was made possible by the generous financial support of the W.M. Keck Foundation. The research is made possible by the data from the Kepler Community Follow-up Observing Program (CFOP). The authors acknowledge all the CFOP users who uploaded the AO and RV data used in the paper. This research has made use of the NASA Exoplanet Archive, which is operated by the California Institute of Technology, under contract with the
National Aeronautics and Space Administration under the Exoplanet Exploration Program. J.W.X. acknowledges support from the National Natural Science Foundation of China (grant No. 11333002 and 11403012), the Key Development Program of Basic Research of China (973 program, Grant No. 2013CB834900) and the Foundation for the Author of National Excellent Doctoral Dissertation (FANEDD) of PR China. J.W. acknowledges the travel fund from the Key Laboratory of Modern Astronomy and Astrophysics (Nanjing University).

\section{REFERENCES}

Adams, E. R., Ciardi, D. R., Dupree, A. K., et al. 2012, AJ, 144, 42 Brandl, B., Hayward, T. L., Houck, J. R., et al. 1997, Proc. SPIE, 3126, 515

Burke, C. J., Bryson, S. T., Mullally, F., et al. 2014, ApJS, 210, 19

Burruss, R. S., Dekany, R. G., Roberts, J. E., et al. 2014, Proc. SPIE, 9148, 914827

Campante, T. L., Barclay, T., Swift, J. J., et al. 2015, ApJ, 799, 170

Davies, R., \& Kasper, M. 2012, ARA\&A, 50, 305

Duquennoy, A., \& Mayor, M. 1991, A\&A, 248, 485

Eggenberger, A., Udry, S., Chauvin, G., et al. 2011, in IAU Symp. 276, The Astrophysics of Planetary Systems: Formation, Structure, and Dynamical Evolution, ed. A. Sozzetti, M. G. Lattanzi, \& A. P. Boss (Cambridge: Cambridge Univ. Press), 409

Giguere, M. J., Fischer, D. A., Howard, A. W., et al. 2012, ApJ, 744, 4

Gilliland, R. L., Cartier, K. M. S., Adams, E. R., et al. 2015, AJ, 149, 24

Han, E., Wang, S. X., Wright, J. T., et al. 2014, PASP, 126, 827

Hayward, T. L., Brandl, B., Pirger, B., et al. 2001, PASP, 113, 105

Horch, E. P., Howell, S. B., Everett, M. E., \& Ciardi, D. R. 2012, AJ, 144, 165

Horch, E. P., Howell, S. B., Everett, M. E., \& Ciardi, D. R. 2014, ApJ, 795, 60

Huber, D., Aguirre, V. S., Matthews, J. M., et al. 2014, ApJS, 211, 2

Jurić, M., \& Tremaine, S. 2008, ApJ, 686, 603

Kane, S. R., Ciardi, D. R., Gelino, D. M., \& von Braun, K. 2012, MNRAS, 425,757

Latham, D. W., et al. 2011, ApJL, 732, L24

Law, N. M., et al. 2014, ApJ, 791, 35

Lillo-Box, J., Barrado, D., \& Bouy, H. 2012, A\&A, 546, A10

Lillo-Box, J., Barrado, D., \& Bouy, H. 2014, A\&A, 566, A103

Lissauer, J. J., Ragozzine, D., Fabrycky, D. C., et al. 2011, ApJS, 197, 8

Lissauer, J. J., Marcy, G. W., Rowe, J. F., et al. 2012, ApJ, 750, 112

Lissauer, J. J., Marcy, G. W., Bryson, S. T., et al. 2014, ApJ, 784, 44

Morton, T. D., \& Winn, J. N. 2014, ApJ, 796, 47

Ngo, H., Knutson, H. A., Hinkley, S., et al. 2015, ApJ, 800, 138

Raghavan, D., McAlister, H. A., Henry, T. J., et al. 2010, ApJS, 190, 1

Roell, T., Neuhäuser, R., Seifahrt, A., \& Mugrauer, M. 2012, A\&A, 542, A92

Rowe, J. F., Bryson, S. T., Marcy, G. W., et al. 2014, ApJ, 784, 45

Touma, J. R., \& Sridhar, S. 2015, Natur, 524, 439

Tremaine, S., \& Dong, S. 2012, AJ, 143, 94

Wang, J., Fischer, D. A., Horch, E. P., \& Xie, J.-W. 2015, ApJ, 806, 248

Wang, J., Fischer, D. A., Xie, J.-W., \& Ciardi, D. R. 2014a, ApJ, 791, 111

Wang, J., Xie, J.-W., Barclay, T., \& Fischer, D. A. 2014b, ApJ, 783, 4

Winn, J. N., \& Fabrycky, D. C. 2015, ARA\&A, 53, 409

Wizinowich, P. L., Acton, D. S., Lai, O., et al. 2000, Proc. SPIE, 4007, 2

Wright, J. T., Fakhouri, O., Marcy, G. W., et al. 2011, PASP, 123, 412

Xie, J.-W., Wu, Y., \& Lithwick, Y. 2014, ApJ, 789, 165 